\title{
EVOLUTIONARY COMPUTING BASED NEURON -COMPUTATIONAL MODEL FOR MICROSTRIP PATCH ANTENNA DESIGN OPTIMIZATION
}

\author{
Rohini Saxena, Mukesh Kumar and Shadman Aslam \\ Department of Electronics and communication, University of Allahabad, U.P., India
}

\begin{abstract}
In this paper, a novel Evolutionary Computing named Adaptive Genetic Algorithm (AGA) based ANN model is developed for rectangular MPA (Microstrip patch antenna). Considering at-hand and Nextgeneration Ultra wideband application demands, the emphasis has been made on retaining optimal lowcost design with desired cut-off frequency. The proposed method employs multiple sets of theoreticallydriven training instances or patch antenna design parameters which have been processed for normalization and sub-sampling to achieve a justifiable and reliable sample size for further design parameter prediction. Procedurally, the input design parameters were processed for normalization followed by sub-sampling to give rise to a sufficient set of inputs to perform knowledge-driven (designparameter) prediction. Considering limitations of the major at-hand machine learning methods which often undergo local minima and convergence while training, we designed a state-of-art new Adaptive Genetic Algorithm based neuro-computing model (AGA-ANN), which helped to predict the set of optimal design parameters for rectangular microstrip patch antenna. The predicted patch antenna length and width values were later used for verification which achieved the expected frequency. The depth analysis revealed that a rectangular patch antenna with width $14.78 \mathrm{~mm}$, length $11.08 \mathrm{~mm}$, feed-line $50 \Omega$ can achieve the cut-off frequency of $8.273 \mathrm{GHz}$, which can be of great significance for numerous UWB applications.
\end{abstract}

\section{KEYWORDS}

Microstrip Patch Antenna, Design Optimization, Evolutionary Computing, Neural Network, UltraWideband.

\section{INTRODUCTION}

The exponential rise in technologies has broadened the horizon for both socio-scientific as well as industry stakeholders to achieve more efficient solutions to meet contemporary demands. Amongst the major innovations, communication systems, and allied antenna technologies have also been the broad region for researchers, where with an increase in demands and operating complexities the solution requires better design and performance. Considering antenna technologies which play an inevitable role in wireless applications, numerous design or models have been proposed [1]. Microstrip Patch Antenna (MPA) is one of the most sought innovations towards wireless applications or transmission systems. Light weight nature, cost-efficient, and ease-of-integration with electronic components make MPA a well-sought antenna model [1-4]. On the other hand, ease-of-fabrication with low-cost substrate materials makes it a popular and significant antenna solution for numerous applications [2][3]. Furthermore, MPA exists in both planers as well as a non-planer surface that enables it to be used in numerous applications including radiofrequency and satellite, defense communication devices, remote sensing, Radars, 
and commercially used Radio Frequency Identification (RFID) [3][4]. Structurally, initially, MPA was developed for wireless communication with two radiating patch elements, one in rectangular shape while the other as a triangular patch. These two patch elements were interfaced using a distinct patch, also called element step sized. To operate for targeting communication, the far-field radiation characteristics of the antenna can be varied by selecting optimal shapes and sizes. It broadens the scope for further optimization; however getting an optimal MPA design solution by considering multiple dependent factors such as resonant frequency, material, length, height remains a challenging task [5][6][32].

In recent years, the miniaturization in electronic component design or expectation has triggered the scientific community to design MPA with low size while retaining high-frequency applications such as Ultra-Wideband (UWB) applications [6]. However, improper or random size-reduction might lead the shift in the resonant frequency and bandwidth that consequently can impact the overall performance of the UWB systems [7]. Therefore, MPA design and allied optimization are must to meet contemporary demands (low size and high frequency) [6][7]. In general, there are two different types of approaches applied for MPA design optimization, Cavity method and Full wave method, which are also called analytical and numerical methods, respectively [8]. Typically, the cavity method, also called the analytical method performs better, especially in terms of analysis [8]. However, both analytical, as well as numerical, have low controllability over operating frequency and bandwidth, which is unfortunately a must in major UWB applications. Factually, it happens due to random (patch) size reduction and hence shifts in operating frequency towards higher size. It also reduced bandwidth and hence can't be suitable for UWB application demands. On contrary, the majority of the classical MPA designs suffer from low bandwidth and reduced gain that confines its suitability for UWB applications [1][810]. Alleviating such issues can be potentially accomplished by designing MPA with optimal substrate thickness, dielectric constants, suitable feeding method, and dimensional values (i.e., patch height and width) [7]. Considering MPA design optimization motive numerous researches have been done; however maintaining an optimal balance between miniaturization and cut-off frequency and bandwidth has remained the challenge, especially for the different UWB applications [7]. Undeniably researches have to lead up to terahertz frequency performance with rectangular MPA; however, their low range confined its suitability for contemporary wireless applications. Noticeably, an optimal solution can be achieved only by considering all design variables together by maintaining an optimal balance between frequency, bandwidth as well as size [7][11][32].

In the last few years, machine learning methods have been applied extensively towards optimization problem, With this motivation, recently authors [12-29] applied Artificial Neural Network (ANN) were made to enhance bandwidth using machine learning methods to obtain optimal patch antenna design with high resonant frequency [18][22][25]. These efforts were made on designing a suitable antenna for circular polarization and annular disk structure. Similarly, a circularly polarized antenna was designed for GPS communication in [30]. However, size and frequency remained an unexplored region for further optimization. ANN is one of the most robust and efficient learning methods can undeniably is suitable to learn over the different design parameters of the MPA and achieve a suitable design solution [12-29]. However, training over a large non-linear data often confines the efficacy of ANN to yield an optimal solution. Local minima and convergence are the key challenges that confine ANN to give optimal solutions. On the other hand, considering multiple design parameters of MPA for optimal design while maintaining high cut-off frequency and bandwidth is a non-convex problem, this has not yet deal with. Considering major limitations of the existing classical approaches, in this paper, a highly robust Evolutionary Computing (EC) assisted ANN model is developed for MPA design optimization to suit UWB application purposes [31][32]. 
International Journal of Computer Networks \& Communications (IJCNC) Vol.13, No.3, May 2021

Hypothesizing the fact that the optimization of a patch antenna design parameters can enable optimal antenna performance for the desired cut-off frequency with higher coverage, in this paper the key emphasis is made on optimizing the design parameter(s) of the microstrip patch antenna. More specifically, this research paper focuses on optimizing the length, width, and other design constraints of the rectangular patch antenna to achieve desired cut-off frequency for UWB applications. Unlike the majority of the classical knowledge-driven and machine learning-based approaches, we focused on enhancing both the data model as well as the computing environment so that the proposed model can deliver optimal design parameters with higher reliability (hypothesizing higher accuracy with minimum error performance). To achieve it, at first design parameter optimization, also called synthesis was performed where inputting substrate height, dielectric coefficients, and cut-off frequency, our proposed AGA-ANN model predicted the optimal values of strip length and width. To assess whether the retrieved length and width values can deliver expected cut-off frequency, we performed verification where the estimated patch length, width, dielectric constants were applied as an input of AGA-ANN to estimate possible cut-off-frequency. To assess the efficacy of AGA-ANN-based patch antenna design estimation, other different neuro-computing models (i.e., ANN-Levenberg Marquardt, ANN-Radial Basis Function, ANN-Gradient Descent) were developed and corresponding performances were examined. Amongst the different neuro-computing models, the proposed AGA-ANN was found more accurate with negligible error performance, which affirms its robustness to yield accurate and optimal design parameters. The proposed work identified patch antenna's width as 14.78 $\mathrm{mm}$, length as $11.08 \mathrm{~mm}$, and feed-line of $50 \Omega$ which could achieve the cut-off frequency of $8.273 \mathrm{GHz}$, which can be vital for many UWB applications.

The remaining sections of this manuscript are divided as follows. Section 2 discusses the related work, which is followed by the discussion of the research questions and problem formulation in Section 3. Section 4 presents the overall system implementation and algorithmic discussion. Section 5 presents the overall simulation results, while the overall research conclusion and inferences are discussed in Section 6. References used in this manuscript are presented at the end of the manuscript.

\section{RELATED WORK}

Considering the significance of MPA for contemporary up-surging UWB applications, numerous researches have been done, though a major fraction of efforts focuses on enhancing materials selection, design (shape or size) enhancement to achieve desired frequency, miniaturized design, and higher bandwidth. However, a significant effort also states that material directive constants being a fixed-range value, dimensional optimization can be of utmost significance. In other words, the authors have recommended applying machine learning methods to obtain a suitable set of design parameters for MPA to be used in UWB applications. The following section briefs a few recently made efforts towards optimizing MPA for UWB applications.

As stated above, numerous efforts have been made to assess MPA in the different shapes to gain respective better performance. In this relation, Chakradhar et al [33] developed a compact dumbbell shape MPA for UWB application to be applied over the range of $3.1 \mathrm{GHz}-10.6 \mathrm{GHz}$ bandwidth. The authors primarily focused on achieving suitable values for size, planar profile, required impedance, bandwidth, and radiation pattern to achieve an intended goal. Mishra et al [34] developed a miniaturized W-slot MPA for UWB application; however they focused on applications as short-distance narrow-band communication. Authors could achieve a resonant frequency of $4.4 \mathrm{GHz}$ with a return loss of $36 \mathrm{~dB}$ by retaining a VSWR of less than 2. It can be well suited for wireless USB, a short distance communication environment. Similarly, in [35], Khan et al developed U-shaped reconfigurable dielectric resonator antennas for $5 \mathrm{G}$ applications. The authors stated that their proposed model with permittivity of 10.2, Microstrip feed line of 50 
$\Omega$ and $0.035 \mathrm{~mm}$ height can achieve above-stated performance. Also, to enhance return loss and bandwidth authors suggested stacking the dielectric resonator antenna at the back of the patch that in conjunction with a switch model can work for different frequencies. Slotted MPA was developed by Roy et al [36], where authors applied designed slotted MPA to achieve higher bandwidth to be used in C-band applications. However, the authors planned to achieve the above stated goal by applying a suitable dielectric constant $(\varepsilon=2.4)$ and substrate thickness $(3.17 \mathrm{~mm})$. ANN-based design simulation yields enhanced bandwidth of $5.88 \mathrm{GHz}$ to $8.28 \mathrm{GHz}$. In [37], Motevasselian et al designed circular MPA using a semi-circle Arc projection mechanism. Here, the prime motive was to assess the efficacy of cuboid ridge to reduce the size of the MPA. Hasan et al [38] designed an F -Band Differential MPA to be used for radar application. Additionally, they designed a waveguide model to be applied for the operating environment $7 \mathrm{GHz}$ with a maximum gain of $12.98 \mathrm{dBi}$ at $122 \mathrm{GHz}$. Radavaram et al [39] designed reconfigurable MPA with dual inverted U-slots that in conjunction with two coaxial probes along the patch's resonant length to achieve a frequency range of $1.98-4 \mathrm{GHz}$. Noticeably authors applied coaxial probes to fed current to introduce phase shift. Similarly, Zhang et al [40] developed a Compact MicrostripFed Patch Antenna which was armored with harmonic suppression ability using a radiating patch and non-radiating $\lambda / 4$ resonators. This method also avoided the need for thick substrate and hence was found size efficient. This prototype antenna could operate for $4.9 \mathrm{GHz}$. A similar effort was made by Zang et al [41]; however, as different authors applied dipole antenna, U-shaped patch, slender stub, and parasitic patch, which eventually yielded fractional bandwidth of 3.43-5.3 GHz. A dual-band slim MPA was designed by Jafargholi et al [42] were to design MPA authors applied patch and shorting, centered, and offset pins with the overall size of $30 \times 50 \mathrm{~mm} \mathrm{2}$. It resulted in better antenna matching while providing efficient radiation performance. It achieved a higher frequency band (3570 MHz). Liu et al [43] designed a Circular patch antenna with high gain and low cross-polarization. The authors achieved an impedance bandwidth of 4.25-6.15 GHz at the central frequency of $5.20 \mathrm{GHz}$. A similar effort was made by He et al [44] who designed dual linearly polarized MPA using a Slot-Loaded cross-shaped patch that enabled orthogonally polarized radiating element functional in TM50 mode. The authors suggested the scope of their model to be used for the 5G network. Unlike above-stated approach, Asaadi et al [45] designed a Square Dense Dielectric Patch Antenna using a Holey superstrate that yielded an impedance bandwidth of $30.8 \mathrm{GHz}$. In [46] Gao et al designed low-size dual-band stacked MPA for WLAN and inter-vehicle Communications. The stacked structure enabled dual-band ability and achieved impedance bandwidths from 2.24 to $2.53 \mathrm{GHz}$ in the low band while 5.42 to $5.98 \mathrm{GHz}$ in the high band. Chen et al [47] developed a polarization-reconfigurable high-gain MPA comprising a ring radiation patch, two switches, and six nonmetallic columns, whereby controlling the PIN switches the antenna was operated in three distinct polarized states; linear polarization (LP), one state for left-hand circular polarization (LHCP), and one state for right-hand circular polarization (RHCP). However, the authors could not address design and dimensional optimization objectives.

Considering the above discusses key literature it can be found that the majority of these models primarily focus on either structural design enhancement by shape, supplementary feed-line, or circuit enhancement. Also, in the last few years, a few types of researches have been done by applying machine learning methods to predict optimal or sub-optimal shape, size, or dimensional characteristics. Khan et al [48] applied the ANN algorithm to predict slot-size and inserted airgap to enhance MPA performance. Also, the authors applied Roger's substrate to achieve better bandwidth performance. The neuro-spectral method was applied in [49] Mishra et al obtained a suitable combination of a continuous function and delta functions to derive the spectral domain Green's functions, which yield eventual parameters for MPA design. Kaur et al [50] applied ANN to obtain dimensions of the rectangular MPA. More specifically, the authors applied the Levenberg-Marquardt ANN model; though they merely focused on assessing ANN efficacy by matching observed or learned output with the theoretical estimation. Neeb has et al [51] too applied ANN for MPA design to be used in radar applications. In addition to the ANN model, the 
authors recommended using Particle Swarm Optimization (PSO) for better design estimation. They found their antenna model suitable for $5 \mathrm{GHz}$ applications. Similarly, Singh et al [52] developed ANN-based rectangular MPA parameter estimator where different ANN variants such as Feed Forward Back Propagation Algorithm (FFBPN), Resilient Back-propagation (RPROP), Levenberg-Marquardt (LM), and Radial Basis functions (RBF) were applied distinctly to obtain the resonant frequency of rectangular MPA. Similarly, in [53], Singh et al assessed different ANN for MPA parameter estimation, especially for a coaxial fed $2.4 \mathrm{GHz}$ E-shaped MPA to be used in IEEE $802.11 \mathrm{~b}$ application environment which demands a frequency band of $2.38 \mathrm{GHz}$ $2.455 \mathrm{GHz}$. Noticeably, authors focused on optimizing Resonant Frequency $\left(f_{r}\right)$ Return Loss (S11 parameter), VSWR $(\mathrm{dB})$, and Input Impedance $\left(R_{i n} \Omega\right)$ by inputting two input parameters, $\mathrm{x}$ coordinate, and y- coordinate of the probe feed point. Similar work was done by Nayak et al [54] who applied ANN for MPA design estimation. The authors recommended a rectangular patch configuration with metal mounted over a large metal ground plane to achieve better resonant frequency. In [55], Deshmukh et al applied ANN for optimal parameter estimation of the suspended equilateral triangular MPAs. Their proposed antenna model was tested over the frequency band of 700 to $6000 \mathrm{MHz}$ while varying the substrate thickness in the range of 0.02 to $0.1 \lambda$. However, the eventual outcome revealed that it could work suitably with a higher thickness substrate. Jain et al [56] too focused on stacked patch antenna design optimization; however unlike conventional ANN models, authors applied ANN and PSO that eventually yield dual-band stacked patch antenna working within $2 \mathrm{GHz}$ to $6 \mathrm{GHz}$ bandwidth. Chiou et al [57] applied ANN to optimize the design of the Substrate Integrate Waveguides (SIW) patch antenna for Ku band applications. The authors found their model operating over the Ku microwave band (12-18 GHz) and resonate at $16.10 \mathrm{GHz}$. In [58], Verma et al designed slot-loaded proximity coupled MPA using knowledge-based ANN, which was found operating from 1 to $6 \mathrm{GHz}$ frequency ranges. Considering high-frequency demands and allied UWB applications, Yoo et al. [59] proposed a dual coupled feeding structure-based patch antenna design for $79 \mathrm{GHz}$ automotive radar applications. Similarly, Bondarik et al. [60] designed a gridded parasitic patch stacked microstrip array antenna design for the $60 \mathrm{GHz}$ band. Sun et al. [61] applied for $5.5 \mathrm{GHz}$ applications. However, these approaches could not address the possibility of harmonics at a higher frequency, which as a result could reduce signal quality over transmission [62][70]. To alleviate this problem Liu et al. [62] proposed an Omni-directional patch antenna design, which was found more effective towards harmonics suppression at higher frequency applications. However, major efforts merely focus on higher bandwidth enhancement, rather than the quality of signal construction over the application environment [63]. Though, a few recent works, such as Xu et al. [64] made effort to inculcate low-profile patch array antenna with corporate stacked microstrip and substrate integrated waveguide feeding structure to retain higher bandwidth with better harmonics conditions. Khan et al. [65] focused on a patch antenna design for channel monitoring purposes, where in addition to the bandwidth, the signal quality assessment accuracy too was equally important. Shenet al. [66] suggested miniaturized patch antenna design to cope up with major at hand 5G communication demands. Islam et al [67] though worked for low UWB frequency patch antenna design; however, it focused on retaining IoT-specific band-performance. A similar effort was made by Chemkha et al. in [68]. An interesting effort was made by Hamid et al. [69] who designed a low-cost, miniaturized patch antenna design with resilience towards defected ground effects. However, it could be effective merely for $900 \mathrm{MHz}$ band applications. The author's fabricated an S-formed RMPA utilizing multilayer perceptron (MLP)- ANN for Sband application considering different boundaries like frequency, efficiency, gain, and directivity in [70]. Siddiqui et al. [71] estimated design parameters for Ellipsoidal fractal MSA through ANN. The calculations of transmission capability of the MSA by utilizing MLP and RBF-ANN based algorithm is proposed in [72].Undeniably, a large number of researches have been done towards patch antenna design; however, very few researches address optimality of design parameter and its optimization. A matter of fact is, the optimality of a patch antenna design primarily depends on the design parameters. The above-discussed survey indicates that the design 
International Journal of Computer Networks \& Communications (IJCNC) Vol.13, No.3, May 2021

optimization of a patch antenna is a fundamental need that in sync with an optimal topological and structural design can enable an optimal antenna solution for major UWB applications.

\section{RESEARCH QUESTIONS}

The overall research intends to find out the optimal answers for the following:

RQ1: Can the strategic implementation of multi-parametric synthesis and verification yield a justifiable MPA design solution?

RQ2: Can the use of Evolutionary Computing or heuristic model be effective to enhance ANN for better MPA design solutions?

RQ3: Can the sub-functional components such as Data Normalization, sampling, etc be effective to achieve an optimal MPA design solution under limited and unbalanced/non-linear training data?

In sync with above-presented research question, considering the significance of a novel and robust MPA design for UWB applications, in this paper the predominant emphasis has been made on optimizing the structural parameters to gain desired resonant frequency, cut-off frequency, and bandwidth. Our proposed model can be stated as a knowledge-based approach where ANN has been trained over the different rectangular MPA design parameters, which eventually yields the optimal set of design variables to achieve the above stated objectives. However, unlike conventional ANN learning methods, which often undergo local minima and convergence during training or learning in this paper a highly robust and efficient evolutionary computing model named Adaptive Genetic Algorithm, has been developed for ANN learning optimization. Such heuristic-based ANN model not only avoids saturation problems or local-minima and convergence but also yields the optimal set of rectangular MPA design parameters to get better performance. Additionally, different sub-functional enhancements have been incorporated such as input -normalization that makes the learning environment resilient to any pre-mature saturation or convergence. It enabled optimized design parameter estimation. Once designing the AGA-ANN learning model, it has been applied to perform a dual-phase implementation.

- MPA Synthesis, and

- MPA Design Verification.

In the MPA synthesis model, the key parameters encompassing the cut-off frequencies (lower and upper frequencies, $\mathrm{f}_{1}$ and $\mathrm{f}_{2}$, respectively), thickness, and dielectric constants of the dielectric material have been fed as input that eventually yields the patch dimensions (i.e., Width and Length) as depicted in Figure 1. As shown in Figure 1, we feed the height of the substrate, cut-off frequency, and dielectric constants as input to be trained by our proposed AGA-ANN model, which yields the width and height of the patch antenna (Figure 4). This process is called synthesis. Once estimating the patch's length and width values, we feed these values to the AGAANN for verification, whether it delivers desired cut-off frequency. As depicted in Figure 2, the proposed AGA-ANN learning model generates the best possible cut-off frequency by the selected patch antenna design parameters. This process is called verification. Considering the overall process of the aforesaid design parameter estimation problem, the key role is being played by the machine learning model (here, AGA-ANN), and therefore we focus on enhancing the neurocomputing environment with a metaheuristic algorithm named AGA, which strengthens ANN to deliver an optimal set of design parameters without undergoing computational adversaries like local minima and convergence. This approach intends to achieve optimal design estimation accuracy with higher reliability. The use of adaptive genetic parameters (i.e., crossover and mutation probability) strengthens AGA-ANN to have low search space and better computational 
efficiency to yield optimal design parameters. Thus, the use of AGA-ANN intends to perform optimal learning over the design parameters (while avoiding NP-hard issues) to yield an optimal set of patch dimensions to have desired resonant frequency and allied MPA performance.

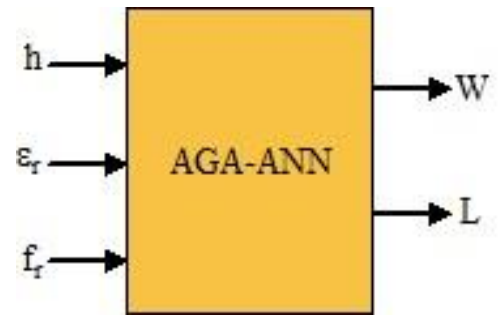

Figure1. MPA synthesis and patch dimensions estimation

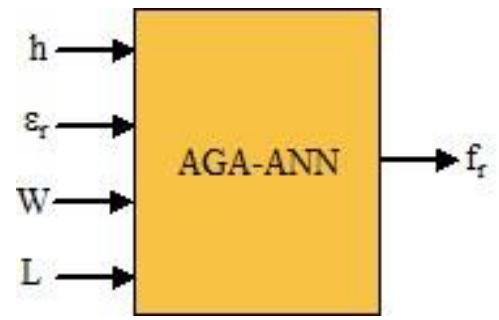

Figure 2. Cut-off frequency verification with obtained patch dimensions

Unlike classical approaches were to verify the obtained results for better antenna performance theoretical models were applied, in this research we have applied the AGA-ANN learning method. In our proposed method, inputting the obtained optimal set of patch dimensions along with the dielectric constants we obtain the desired cut-off frequency, signifying the performance of the proposed MPA model. Additionally, to assess the efficacy of the proposed model, the results are examined in terms of statistical performance measures like accuracy, error profile, etc. A detailed discussion of the overall proposed model is given in the subsequent sections.

\section{PRoposed SYSTEM}

This section primarily discusses the overall proposed system for the rectangular MPA design optimization. The overall research model encompasses two key phases, MPA synthesis, and $\mathrm{z}$ MPA verification; however, to enable optimal computation in this process different procedural enhancements have been done. Considering the overall implementation paradigm, we define this research as following sequential phases.

- Rectangular MPA Parameter Definition

- MPA Data Generation

- Data Normalization

- MPA Synthesis

- ANNs Based MPA dimensional parameter estimation (i.e., L and W estimation)

- AGA-ANN based MPA dimensional parameter estimation

- MPA Design verification (for cut-off frequency or resonant frequency).

- ANNs Based MPA Design verification.

- AGA-ANN based MPA Design verification.

\subsection{Rectangular MPA: The Parameter Definition}

Considering the parametric definition and estimation for the rectangular MPA model before discussing the proposed model and its implementation, it can be vital to understand a snippet of the design considered and the allied mathematical model involved. With this motive, a brief of the rectangular MPA design and allied parametric definition is given in the subsequent sections. As stated, we intend to obtain optimized design parameters for rectangular MPA. Here, we have considered the substrate dielectric constants in the range of 2.3 to 12 ; however, we hypothesize the dielectric substrate to be thicker. Additionally, the dielectric constant is considered to be less to achieve wide bandwidth and efficiency. An illustration of the considered rectangular MPA 
International Journal of Computer Networks \& Communications (IJCNC) Vol.13, No.3, May 2021

design is given in Figure 3. Established on a simplified formulation for the rectangular MPA model has been described [4].

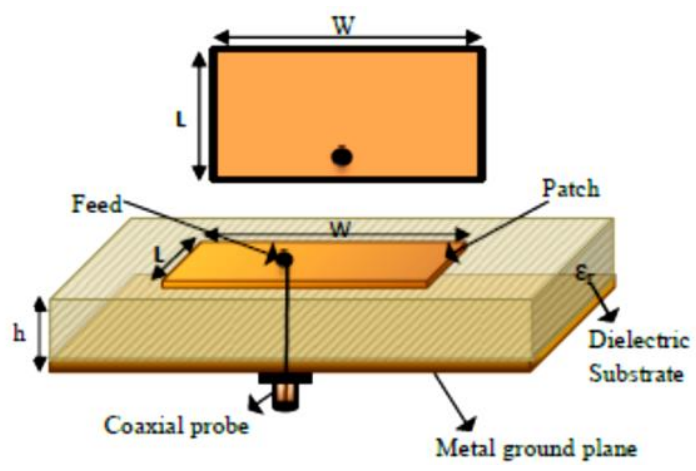

Figure 3. Rectangular MPA model

The width $(\mathrm{w})$ for the effective radiator and its respective dielectric constant $\left(\varepsilon_{\text {eff }}\right)$ have been obtained using equation (1) and (2), where $C$ is the velocity of the light.

$$
\begin{gathered}
w=\frac{C}{2 f_{0}} \sqrt{\frac{2}{\varepsilon_{r}+1}} \\
\varepsilon_{e f f}=\frac{\varepsilon_{r}+1}{2}+\frac{\varepsilon_{r}-1}{2}\left[1+12 \frac{h}{W}\right]^{-\frac{1}{2}}
\end{gathered}
$$

The length of the patch $\mathrm{L}$ is obtained using equation (3-4) and effective length can be obtain through using equation (5).

$$
\begin{gathered}
L=L_{e f f}-2 \Delta L \\
L=\frac{1}{2 f_{r} \sqrt{\varepsilon_{e f f}} \sqrt{\mu_{0} \varepsilon_{r}}}-2 \Delta L \\
L_{e f f}=\frac{C}{2 f_{00} \sqrt{\varepsilon_{e f f}}}
\end{gathered}
$$

Noticeably, in equation (4), the parameter $\Delta \mathrm{L}$ signifies the increase in the electric length, which is usually caused due to the fringing nature of patch. Mathematically, $\Delta \mathrm{L}$ is obtained using equation (6).

$$
\Delta L=0.412 h\left(\frac{\left(\varepsilon_{e f f}+0.3\right)\left(\frac{w}{h}+0.264\right)}{\left(\varepsilon_{e f f}-0.258\right)\left(\frac{w}{h}+0.813\right)}\right)
$$

\subsection{MPA Data Generation}

Being a knowledge-driven model, ANN and/or AGA-ANN requires training instances or parameters to obtain the eventual design solution. Considering this fact, we obtained a set of input instances by considering the UWB application environment. Observing the UWB 
International Journal of Computer Networks \& Communications (IJCNC) Vol.13, No.3, May 2021

application environment such as Wireless Local Area Network (2.4-2.48 GHz and 5.75-5.825 $\mathrm{GHz})$, Inter-vehicular communications (5.85-5.925 GHz), etc, we simulated different training instances with the resonant frequencies in UWB ranges. At first considering the resonant frequency of $4.99 \mathrm{GHz}$, with the dielectric constant of 4.4 (though it can vary in between 2.2 to 12), the height of dielectric material was maintained at $1.6 \mathrm{~mm}$. With these initial parameters the dimension of the patch was obtained using the above discussed mathematical equations (1-6) for $4.99 \mathrm{GHz}$ resonant frequency, and thus the Patch Length and Width values were obtained as $13.34 \mathrm{~mm}$ and $17.7 \mathrm{~mm}$, respectively. Noticeably, similar to [34] and [37], we introduced a $50 \Omega$ port connected to the patch using the Microstrip fed-line method. To be noted, feed signifies the specific point where the patch radiates the maximum. In our proposed model, we applied equation (7) to estimate the width of the Microstrip feed line, where $\varepsilon_{\text {eff }}$ signifies the effective (substrate) dielectric constant. Mathematically,

$$
Z_{0}=\left(\frac{120 \Pi}{\left[\sqrt{\left(\varepsilon_{e f f}\right)}\left\{\frac{w_{0}}{h}+1.393+0.667 \ln \left(\frac{w_{0}}{h}+1.444\right)\right\}\right]}\right)
$$

The above mathematical model for the aforementioned initial parameters gives $w_{0}=2.94 \mathrm{~mm}$. Thus, obtaining the above-stated parameter different simulations and allied assessments were made for the return losses and frequency. Varying the patch dimensions, different resulting parameters were obtained to prepare the training instances. Considering application-specific environment, the lower cut-off frequency $\left(f_{l}\right)_{\text {was }}$ maintained at $4.94 \mathrm{GHz}$, while $5.95 \mathrm{GHz}$ was maintained as the upper cut-off frequency $\left(f_{u}\right)$. Similarly, we varied the dimensional parameters of the patch, while retaining the other parameters as constant and vice-versa. Thus, it helped to obtain a few samples needed to be used for MPA synthesis using ANN and further enhanced AGA-ANN models.

\subsection{Data Normalization}

Observing the input data nature the width and length are in millimeter $(\mathrm{mm})$, while frequency ranges are in GHz. Similarly, the dielectric constant values are in tens. Such non-linearity and imbalance nature of data can force any machine learning model including ANN to undergo saturation and/or local minima and convergence. Interestingly, many researchers have been done for MPA parameter estimation or antenna design optimization; however, no significant effort is made to address such parametric nonlinearity or imbalance issues. Considering this fact, in this research paper, we have applied the "Data Normalization" concept over the obtained input training samples for MPA design optimization. This approach maps overall input data in the range of $[0,1]$ and hence avoids any probability of premature convergence or saturation. In our proposed model, we applied the Min-Max Normalization algorithm that maps or normalizes input data such as frequency, length, width, and dielectric constant in the range of $[0,1]$. Here, each data element $\mathrm{x}_{\mathrm{i}}$ of the MPA design parameter $\mathrm{X}$ is mapped to the allied normalized value $\mathrm{x}_{\mathrm{i}}^{\prime}$ in the range of $[0,1]$ using equation (8).

$$
\operatorname{Normalized}\left(x_{i}\right)=x_{i}^{\prime}=\frac{x_{i}-\min (X)}{\max (X)-\min (X)}
$$

Where, component $\min (\mathrm{X})$ and $\max (\mathrm{X})$ signifies the minimum and maximum values of $\mathrm{X}$ correspondingly. 


\subsection{MPA Synthesis}

Once normalizing the input design parameters, it was fed as input to the ANN models, followed by the AGA-ANN algorithm. The detailed discussion of the proposed ANN variants including GD-ANN, ANN-RBF, ANN-LM, and eventually our proposed AGA-ANN model is given in the subsequent sections.

\subsubsection{ANN Design for MPA Synthesis}

Amongst the major machine learning models for data analysis and complex problem-solving tasks, ANN has emerged as a dominating solution. ANN mimics the behavior of the human brain, where it learns over the existing patterns to classify further unknown entities or variables. The suitability of ANN has made it a common machine learning model to be used in major Artificial Intelligence (AI) computations. Functionally, ANN comprises multiple neurons carrying input data or signals to be processed at the different hierarchies including the input layer, hidden layer and delivers the outcome at the output layer. Noticeably, ANN implements an error reduction principle to perform learning over the input data, where it estimated the difference between the expected value and the observed value, commonly called error. ANN intends to reduce this error iteratively to achieve minimum or zero error and thus classifies data into the most relevant class or classes. However, in this process obtaining optimal classification or in other, words error reduction requires suitable learning methods to achieve objective function (i.e., zero error) without forcing the algorithm to undergo saturation or convergence. To achieve it, ANN has undergone numerous evolutions including SD-ANN, GD-ANN, GDX-ANN, ANN-BP, ANNRBF, ANN-LM, and many more. Even in the last few years heuristic based ANNs have been proposed to handle large-scale data which might require swift learning and high accuracy. Enhancements have been made especially to enhance overall learning and weight estimation activities. As depicted in Figure 4, the prime efforts have been made on optimizing the values of weights $\left(\mathrm{W}\right.$ and $\mathrm{W}_{\mathrm{k}}$ ). Some of the key variants of ANN are, Steepest Descent ANN (SD-ANN), Gauss-Newton-ANN (GN-ANN), Gradient Descent ANN (GD-ANN), Levenberg-Marquardt ANN (LM-ANN), Radial Basis Function ANN (RBF-ANN), etc, which primarily differ in terms of the manner it learns and updates its learning weights to achieve optimal performance. However, amongst these approaches, GD-ANN, LM-ANN, ANN-GDX (also called adaptive learning-based ANN) and RBF-ANN have been explored extensively due to their ability to process a large set of input data in low computational time. Considering it as motivation, in addition to the proposed AGA-ANN model, we have applied different ANN variants to perform MPA synthesis, where we feed different cut-off frequency parameters, dielectric constant, and height of the dielectric material as input to obtain the optimal (corresponding) values of the patch's width and length.

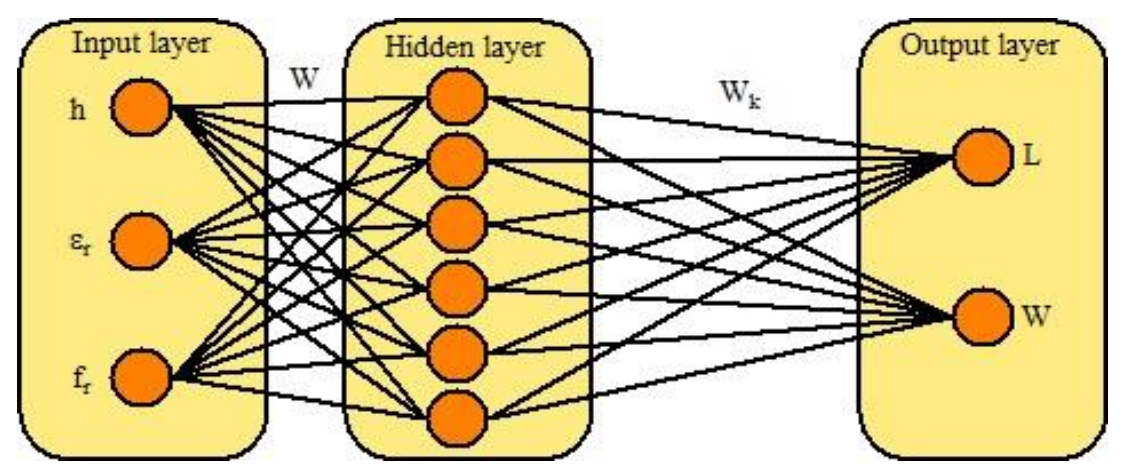

Figure 4. ANN based MPA synthesis 
International Journal of Computer Networks \& Communications (IJCNC) Vol.13, No.3, May 2021

As depicted in Figure 4 a conventional ANN model encompasses three layers, input layer, hidden layer, and output layer. In our proposed MPA design optimization model, dielectric constants, frequency, and height of the substrate are fed as input to the input layer (Figure 4). Precisely stating, in our proposed ANN models, three inputs were feed as input-neurons. Here, we have applied a linear activation function at the input layer that enables output of the input layer equal to the input of the input layer (i.e., $\mathrm{O}_{\mathrm{o}}=\mathrm{I}_{\mathrm{i}}$ ). Similarly, at the hidden layer sigmoid function has been applied that results $O_{h}$. Here, for MPA synthesis we assigned two output neurons, as we intend to obtain $\mathrm{W}$ and $\mathrm{L}$ parameters as a learning result. Noticeably, with the input of the hidden layer $\left(I_{h}\right)$, we obtained the output $\mathrm{O}_{h}$ using the sigmoid function equation (9).

$$
O_{h}=\frac{1}{1+e^{-I_{h}}}
$$

Thus, the final result obtained after ANN learning is the value of $\mathrm{O}_{\mathrm{o}}=\frac{1}{1+\mathrm{e}^{-\mathrm{O}_{\mathrm{i}}}}$. Typically, the ANN model is expressed as a function $Y^{\prime}=f(W, X)$ where $Y^{\prime}$ states the resultant vector, while Wand $\mathrm{X}$ signify the weight value and the input vector, correspondingly. To achieve the optimal value of the Weight and Length of the patch, the ANNs intend to reduce an error value iteratively. Here, we applied Mean Square Error (MSE) as the error function to obtain the optimal design parameters or equation (10). In equation (10), y states the observed value, while $y_{i}^{\prime}$ signifies the expected result at an iteration. Also, we assigned learning rate for

$$
M S E=\frac{1}{n} \sum_{i=1}^{n}\left(y_{i}^{\prime}-y_{i}\right)^{2}
$$

ANNs (excluding AGA-ANN) as 0.01, while the error or stopping condition was fixed as 0.001 . It means the ANN would keep on iterating till achieving the error of 0.001 . As depicted in the above equation, ANN intends to learn over the input four parameters of patch antenna; however, learning while reducing error plays a significant role in the overall accuracy and reliability of the learning model. Different ANN variants can have distinct performance. Considering this fact, in this paper we have applied different ANN variants and their respective performance is examined. A brief of the different ANN algorithms applied is given in the subsequent sections.

\subsubsection{GD-ANN}

As already stated, ANN intends to reduce error function iteratively for all training sets. Let regression for the learning be

$$
\begin{gathered}
w^{*}=\underset{w}{\operatorname{argmin}} L(w) \\
L(w)=\sum_{t=1}^{N} L\left(y_{t}, f_{w}\left(x_{t}\right)\right)+\lambda R(w)
\end{gathered}
$$

As depicted in equation (11), where $\mathrm{f}_{\mathrm{w}}(\mathrm{x})$ is non-linear in the weight vector $(\mathrm{w})$, ANN-GD intends to achieve a local optimum for equation (11) using GD algorithm. Here, GD updates w iteratively by replacing $\mathrm{w}_{\mathrm{t}}$ by $\mathrm{w}_{\mathrm{t}+1}$ using equation (13-14).

$$
\begin{gathered}
\mathrm{w}_{\mathrm{t}+1}=\mathrm{w}_{\mathrm{t}}-\eta_{\mathrm{t}} \nabla \mathrm{L} \\
\mathrm{w}_{\mathrm{j}, \mathrm{t}+1}=\mathrm{w}_{\mathrm{j}, \mathrm{t}}-\eta_{\mathrm{t}} \frac{\partial \mathrm{L}}{\partial \mathrm{w}_{\mathrm{j}}}
\end{gathered}
$$


International Journal of Computer Networks \& Communications (IJCNC) Vol.13, No.3, May 2021

In equation (13-14), $\eta_{\mathrm{t}}$ represents the learning rate that usually decreases with $t$. Here, $\nabla \mathrm{L}$ states the error, $\frac{1}{n} \sum_{i=1}^{n}\left(y_{i}^{\prime}-y_{i}\right)^{2}$.

\subsubsection{RBF-ANN}

Similar to the traditional ANN model, a Radial Basis Function (RBF) ANN too possesses an input layer, a hidden layer, and an output layer; however, the neurons in the hidden layer comprise Gaussian transfer functions whose results are inversely proportional to the distance from the center of the neuron. On the other hand, functionally, RBF-ANN is similar to the KMeans clustering methods and Probabilistic Neural Network (PNN). The predominant difference is that the other models as stated (PNN) possess a single neuron for each data-point in the training set, while RBF-ANN contains multiple neurons but less than the number of training points. Undeniably, for medium-size datasets, PNN is sufficient; however, as targeted in current BigData analytics purpose where there is a huge dataset RBF-ANN seems a potential solution. With this motivation, in this paper, we have applied RBF-ANN as one of the base classifiers to estimate the patch dimensional parameters (i.e., W and L). Functionally, in RBF-ANN the hidden units enable the certain set of functions consisting of a random basis for the input patterns. Here, the hidden units are stated as radial centers and are signified as vector $c_{1}, c_{2}, \ldots, c_{h}$. In this method, the input variables (say, input space) are transferred into hidden space using a non-linear transformation approach, while the transformation from the hidden unit space to the output space is linear dimension of each center for an $\mathrm{n}$ point input network (i.e., $\mathrm{n} \times 1$ dimension). Here, the RBF in the hidden layer generates a non-zero response and each hidden unit possesses its receptive field in input space. For an input vector $x_{i}$ existing in the receptive field for center $c_{j}$, activate $c_{j}$ by selecting suitable weights to achieve the intended target output. Mathematically, the target output is defined as equation (15)

$$
\mathrm{y}^{\prime}=\sum_{\mathrm{j}=1}^{\mathrm{h}} \emptyset_{\mathrm{j}} \mathrm{w}_{\mathrm{j}}, \quad \emptyset_{\mathrm{j}}=\emptyset\left(\left\|\mathrm{x}-\mathrm{c}_{\mathrm{j}}\right\|\right)
$$

In equation (15), $\mathrm{w}_{\mathrm{j}}$ represents the weight of the jth center, while $\emptyset$ states radial function, PseudoInverse, or hybrid. Considering the popularity of the GD algorithm we have applied it as the activation function. To achieve optimal classification, RBF-ANN requires optimal selection of the weight vector $w_{j}$ and $c_{j}$, where $j=1, \ldots, h$. In this paper, we have applied GD based weight update method for RBF-ANN learning over the job datasets and user's profile variable. In addition to the GD-ANN and RBF-ANN, we have applied the LM-ANN model as well to estimate the optimal dimensional parameters for patch antenna. A snippet of LM-ANN is given as follows:

\subsubsection{LM-ANN}

Unlike conventional GD and Steepest Descent (SD) ANN learning algorithms, Levenberg Marquardt ANN (LM-ANN) iteratively performs localization of the minimum value of the multivariate function, which is often called the Sum of Squares (SoS) of the non-linear realvalued functions. Unlike above stated NN models, LM-ANN performs swift weight update when learning and thus achieves computationally as well as time-efficient. Also, LM-ANN combines the robustness of both SD-ANN and GD-ANN algorithms, where selecting the learning rate, achieves objective function swiftly. The weight-update function of LM-ANN is given by equation (16).

$$
\mathrm{W}_{\mathrm{j}+1}=\mathrm{W}_{\mathrm{j}}-\left(\mathrm{J}_{\mathrm{j}}^{\mathrm{T}} \mathrm{J}_{\mathrm{j}}+\mu \mathrm{I}\right)^{-1} \mathrm{~J}_{\mathrm{j}} \mathrm{e}_{\mathrm{j}}
$$


International Journal of Computer Networks \& Communications (IJCNC) Vol.13, No.3, May 2021

$$
J=\left[\begin{array}{cccc}
\frac{d}{d W_{1}}\left(E_{1,1}\right) & \frac{d}{d W_{2}}\left(E_{1,1}\right) & \cdots & \frac{d}{d W_{N}}\left(E_{1,1}\right) \\
\frac{d}{d W_{1}}\left(E_{1,2}\right) & \frac{d}{d W_{2}}\left(E_{1,2}\right) & \cdots & \frac{d}{d W_{N}}\left(E_{1,2}\right) \\
\vdots & \vdots & & \vdots \\
\frac{d}{d W_{1}}\left(E_{P, M}\right) & \frac{d}{d W_{2}}\left(E_{P, M}\right) & \cdots & \frac{d}{d W_{N}}\left(E_{P, M}\right)
\end{array}\right]
$$

In equation (16), $\mathrm{W}_{\mathrm{j}}$ signifies the current weight, while $\mathrm{W}_{\mathrm{j}+1}$ states the updated weight. The variable I presents the identity matrix, and J presents the Jacobian matrix. The parameter $\mu$ presents the combination coefficient where the low value of $\mu$ makes it functional as GD-ANN, while high value forces it to behave as SD-ANN. Equation (17) presents the mathematical expression for the Jacobian matrix used. Where, $\mathrm{N}$ states the total weights, $\mathrm{P}$ presents the input pattern and the output pattern is shown by M. As already stated, the proposed LM-ANN model encompasses the efficacy of both SD-ANN as well as GD-ANN, where varying the value of $\mu$, as given in equation (16), it can be operated as both SD-ANN as well as GD-ANN. A snippet of the overall process involved in our proposed LM-ANN model is given in Figure 5.

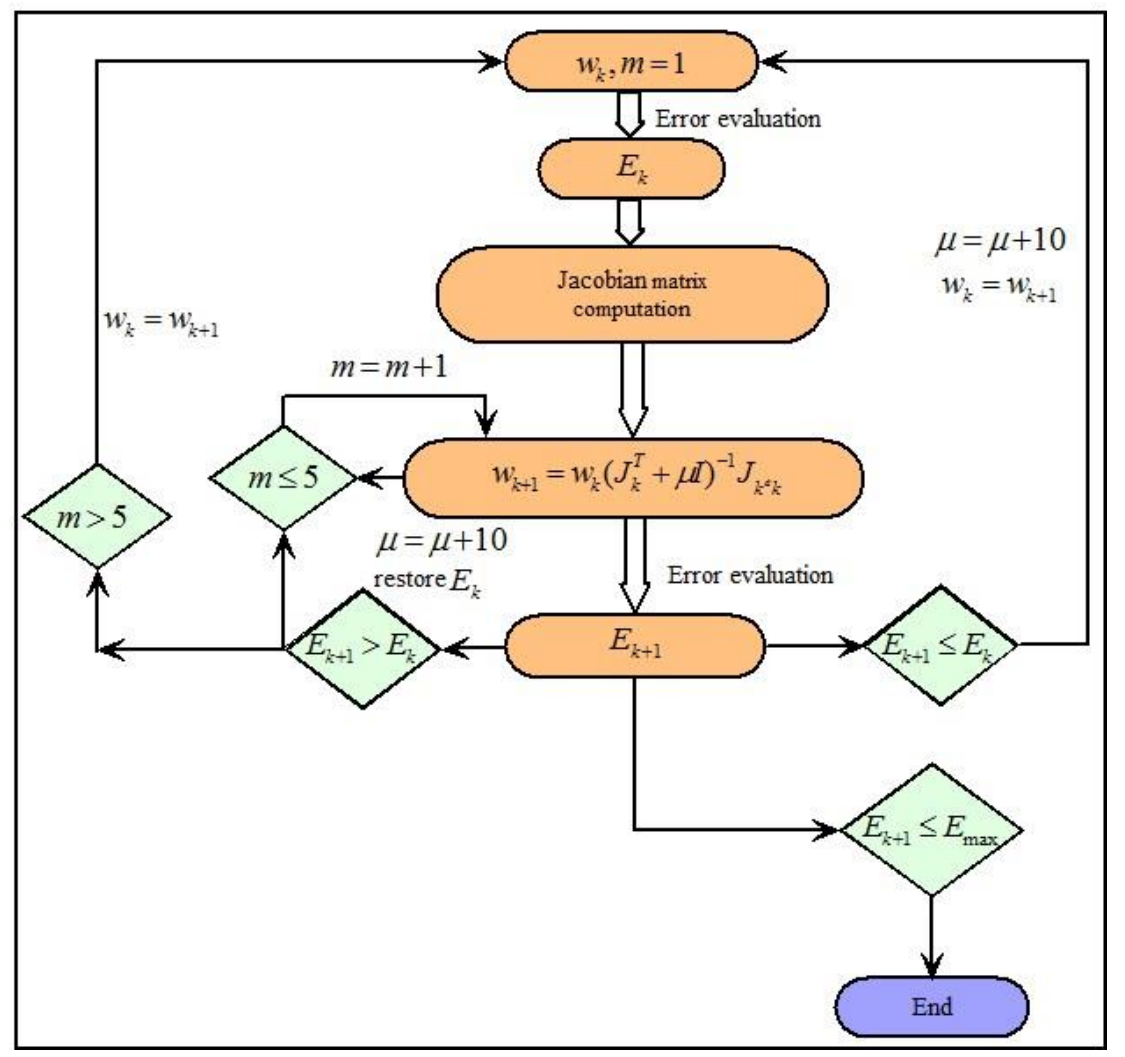

Figure 5. Proposed LM-ANN based learning for patch antenna design parameter estimation.

As depicted in Figure 5, for each output pattern $m$, the proposed model at first estimates the error value, which is subsequently processed for Jacobian estimation as given in (17). Thus, obtaining the Jacobian matrix, we applied equation (16) to estimate subsequent weight values to reduce the error $E_{K+1}$. Noticeably, assigning the higher value of $\mu$ enables it to function as SD-ANN, while a lower value of $\mu$ enables the same to behave as GD-ANN. As depicted in Figure 5, once finding the estimated error $E_{K+1}<E_{K}$, the proposed LM-ANN model updated the learning coefficient $\mu$, 
International Journal of Computer Networks \& Communications (IJCNC) Vol.13, No.3, May 2021

which is subsequently used as feedback to update the learning weight parameter. This process continues till the error $E_{K+1}<E_{\text {Max }}$. This state signifies the convergence state where it predicts the targeted class. Noticeably, observing the above discussion it can easily be found that estimation of the weight parameter is a highly iterative task and hence can force the algorithm to undergo local minima and convergence, which can decisively impact the accuracy and reliability of the design parameter estimation. This problem can be even more severe in case of increased search space or the number of weight parameters to be estimated, which is normal in the at-hand patch antenna design parameter estimation. Considering this fact, in this paper we proposed evolutionary computing -assisted ANN learning model. Unlike classical meta-heuristic concepts, in this paper, we designed adaptive genetic algorithm (AGA) based ANN (AGA-ANN). A detailed discussion of the proposed AGA-ANN model is given in the subsequent section.

\subsubsection{AGA-ANN Learning}

Almost all major machine learning algorithms undergo adversaries like local minima and convergence. On the other hand, with more number hidden neurons and huge data sets, it suffers premature convergence and hence exhibits false alarm. Inappropriate edge-weight estimation during learning is the predominant reason for local minima and convergence. Considering this fact, we have exploited EC algorithms to augment learning rate and allied optimal weight estimation. Though, numerous heuristic (EC) approaches are available for sub-optimal solution retrieval, such as Genetic Algorithm (GA), Particle Swarm Optimization (PSO), Ant Colony System (ACS), Cuckoo Search (CS), Artificial Immune System (AIS), Flower Pollination Algorithm (FPA), etc; however, their efficacy often remains confined due to convergence issues, higher computation time and cost. Considering this fact, in this paper unlike native EC algorithms, we have developed an enhanced GA model called AGA that introduces dynamic or adaptive parameter estimation (Crossover probability and mutation probability) and updates. Noticeably, in this paper AGA algorithm has been applied to enhance learning weight estimation of ANN classifier to estimate optimal dimensional parameters of the patch antenna. The detailed discussion of the proposed AGA-ANN model is given as follows:

Extracting the data samples has been fed as input to the AGA-ANN that as a result predicts the values of the cut-off frequency. As depicted in Figure 4, the three patch antenna parameters are fed as input that eventually performs learning and estimates the optimal values of the patch width and length at the output layer. Since our proposed ANN model comprises 3 input neurons and hence we have considered 6 hidden neurons and 2 output neurons. In AGA-ANN linear activation function has been applied at the input layer that provides output equivalent to the input (i.e., $\mathrm{O}_{\mathrm{o}}=\mathrm{I}_{\mathrm{i}}$ ). On the other hand, at the hidden layer sigmoid function has been implemented that outputs $\mathrm{O}_{\mathrm{h}}$. Feeding results of the input layer as the input of the hidden layer $\mathrm{I}_{\mathrm{h}}$, it applies the sigmoid function to generate output as $\mathrm{O}_{\mathrm{h}}$. Mathematically, $\mathrm{O}_{\mathrm{h}}$ is calculated using (9). Observing the overall ANN structure as depicted in Figure 4, a total of 30 weight parameters are required to be updated iteratively in ANN to achieve optimal performance or error reduction. This mechanism with conventional weight update methods is highly complex, and therefore we have applied the AGA algorithm as a heuristic model to obtain the weights iteratively for better and efficient learning. The detailed discussion of the AGA model and eventual AGA-ANN is given as follows:

\subsubsection{Adaptive Genetic Algorithm (AGA)}

Genetic Algorithm (GA) being one of the most efficient and robust heuristic models exploits the concept of adaptive search and Darwin's selection criteria to achieve the sub-optimal solutions. Principally, GA simulates the natural behavior of evolution, especially the principle of Darwinian natural selection. Functionally, GA is started with an initial population where it a generates 
International Journal of Computer Networks \& Communications (IJCNC) Vol.13, No.3, May 2021

random population where each population signifies a unit sub-solution or candidate solution. In fact, these solutions are the chromosomes having a structure of binary strings where the entire parameters are encoded. Generating the initial population, GA estimates the fitness value for each chromosome that refers to certain user-defined function depicting the ability of each chromosome. In other words, higher fitness states a better probability of a chromosome to become a part of the candidate solution in the next generation to provide a more effective solution. Based on the estimated fitness values, children or offspring are generated using GA operators called crossover probability $\left(\mathrm{P}_{\mathrm{c}}\right)$ and mutation probability $\left(\mathrm{P}_{\mathrm{m}}\right)$. In GA-based optimization, the algorithm continues obtaining the best solution over the varied generations to achieve a set of solutions fulfilling expected criteria (here, minimum RMSE). Now, considering our proposed ANN structure (i.e., $\mathrm{i}-\mathrm{h}$ - o) with $\mathrm{i}$ input neurons, $\mathrm{h}$ hidden neurons, and $\mathrm{O}$ output neurons, three patch antenna parameters are fed as input nodes. With this configuration (i.e., $i=3$, $\mathrm{h}=6$ and $\mathrm{O}=2$ ) the total weight component or factors required would be equation (18).

$$
N=(i+O) * h
$$

In AGA-ANN, we consider positive non-zero values as weight solution. Consider, 1 be the length of the gene, then the length of chromosome can be obtained as equation (19).

$$
L_{\text {Chrom }}=N * l=(i+O) * h * l
$$

To estimate fitness value of each chromosome or population, we have derived weight values for each edge-graph, $\mathrm{W}_{\mathrm{k}}$ using equation (20). Mathematically,

$$
W_{k}=\left\{\begin{array}{c}
\text { if } 0 \leq x_{k l+1}<5 \\
-\frac{x_{k l+2 *} 10^{l-2}+x_{k l+3 *} 10^{l-3}+\cdots+x_{(k+1) l}}{10^{l-2}} \\
\text { if } 5<=x_{k l+l}<=9 \\
+\frac{x_{k l+2 *} 10^{l-2}+x_{k l+3 *} 10^{l-3}+\cdots+x_{(k+1) l}}{10^{l-2}}
\end{array}\right.
$$

As stated, in our proposed AGA-ANN model, we have applied MSE as the objective function to retrieve optimal weight solution. The following algorithm depicts the process for fitness value estimation for each chromosome

AGA-ANN Fitness Calculation

Input: Input patch antenna parameters $\bar{I}_{i}=\left(I_{1 i}, I_{2 i}, I_{3 i}, \cdots, I_{l i}\right)$

Output: $\bar{T}_{i}=\left(T_{1 i}, T_{2 i}, T_{3 i}, \cdots, T_{n i}\right)$

Where $\overline{\mathrm{I}}_{\mathrm{i}}, \overline{\mathrm{T}}_{\mathrm{i}}$ represent the input and output pairs of the $\mathrm{i}-\mathrm{h}-\mathrm{o}$ ANN structure.

Step-1 :Estimate weight vector $\overline{\mathrm{W}}_{\mathrm{i}}$ from $\mathrm{C}_{\mathrm{i}}$ using equation (21)

$$
W_{k}=\left\{\begin{array}{c}
\text { if } 0 \leq x_{k d+1}<5 \\
-\frac{x_{k d+2 *} 10^{d-2}+x_{k d+3 *} 10^{d-3}+\cdots+x_{(k+1) d}}{10^{d-2}} \\
\text { if } 5<=x_{k d+d}<=9 \\
+\frac{x_{k d+2 *} 10^{d-2}+x_{k d+3 *} 10^{d-3}+\cdots+x_{(k+1) d}}{10^{d-2}}
\end{array}\right.
$$

Step-2 :Assuming $\overline{\mathrm{W}}_{\mathrm{i}}$ as a fixed weight parameter, execute ANN training function for $\mathrm{N}$ input instances and calculate output $\mathrm{O}_{\mathrm{i}}$

Step-3 :Calculate error $E_{j}$ as the difference of expected value and current 
International Journal of Computer Networks \& Communications (IJCNC) Vol.13, No.3, May 2021

estimated value for each input instance $\mathrm{j}$

$E_{j}=\left(T_{j i}-O_{j i}\right)$

Step-4 :Calculate RMSE of each chromosome $\mathrm{C}_{\mathrm{i}}$ using equation (22)

$$
E_{i}=\sqrt{\frac{\sum_{j=1}^{j=N} E_{j}(\text { Target }- \text { Observed })}{N}}
$$

Where, $\mathrm{N}$ states the total number of training data set.

Step-5 :Calculate the fitness value for chromosome $\mathrm{C}_{\mathrm{i}}$ using equation (23).

$$
F_{i}=\frac{1}{E_{i}}=\frac{1}{\sqrt{\frac{\sum_{j=1}^{j=N} E_{j}}{N}}}
$$

Figure 6. Pseudo code for fitness estimation algorithm.

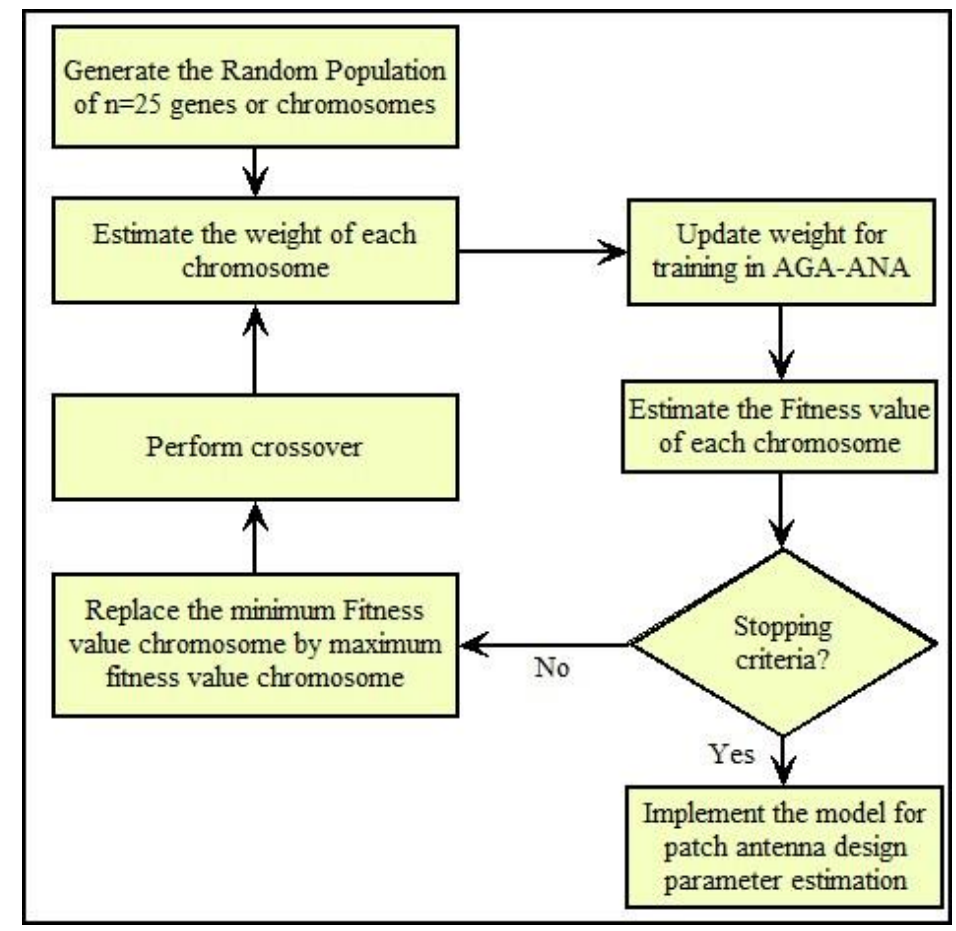

Figure 7. Proposed AGA-ANN based weight estimation

In classical GA algorithms typically a static stopping criterion such as the number of generations or iterations are used. However, such approaches often undergo local minima and convergence due to increased search space and allied computational overheads. Considering this fact, we proposed the AGA-ANN model by applying dynamic or adaptive genetic parameters (i.e., $\mathrm{P}_{\mathrm{c}}$ and $\mathrm{P}_{\mathrm{m}}$ ) that avoid the issue of over-fitting or under-fitting. One of the most efficient and noticeable contributions of our proposed model is that unlike the traditional GA algorithm where the number of iterations is used as stopping criteria, AGA-ANN considers an adaptive update approach. It is supported by the fact that during training, there can be multiple chromosomes having similar fitness value that could force ANN to undergo local minima and convergence. Considering this fact, AGA-ANN estimates the number of chromosomes having a similar fitness value which is further used to update $\mathrm{P}_{\mathrm{c}}$ and $\mathrm{P}_{\mathrm{m}}$ dynamically by the using equation (24). 
International Journal of Computer Networks \& Communications (IJCNC) Vol.13, No.3, May 2021

$$
\begin{gathered}
\left(P_{c}\right)_{k+1}=\left(P_{c}\right)_{k}-\frac{C_{1} * N_{N C F}}{7} \\
\left(P_{m}\right)_{k+1}=\left(P_{m}\right)_{k}-\frac{C_{2} * N_{N C F}}{7}
\end{gathered}
$$

In equation (24), the variables $\left(\mathrm{P}_{\mathrm{c}}\right)_{\mathrm{k}+1}$ and $\left(\mathrm{P}_{\mathrm{m}}\right)_{\mathrm{k}+1}$ signify the updated crossover and mutation probability, while its current probability is given by $\left(\mathrm{P}_{\mathrm{c}}\right)_{\mathrm{k}}$ and $\left(\mathrm{P}_{\mathrm{m}}\right)_{\mathrm{k}}$. Furthermore, we have considered static values for the coefficient parameters $C_{1}$ and $C_{2}$ as 0.1 and 0.01 , respectively. The parameter $\mathrm{N}_{\mathrm{NCF}}$ signifies the total chromosome having a similar fitness value.

\subsection{MPA Design verification}

Similar to the MPA synthesis, we have applied ANN variants to perform verification, where height, length, width, and substrate dielectric constants are fed as input; the output is obtained as the cut-off frequency. Noticeably, the predominant intend is to assess whether the obtained (optimal) value of the width and length achieves desired cut-off frequency or not.

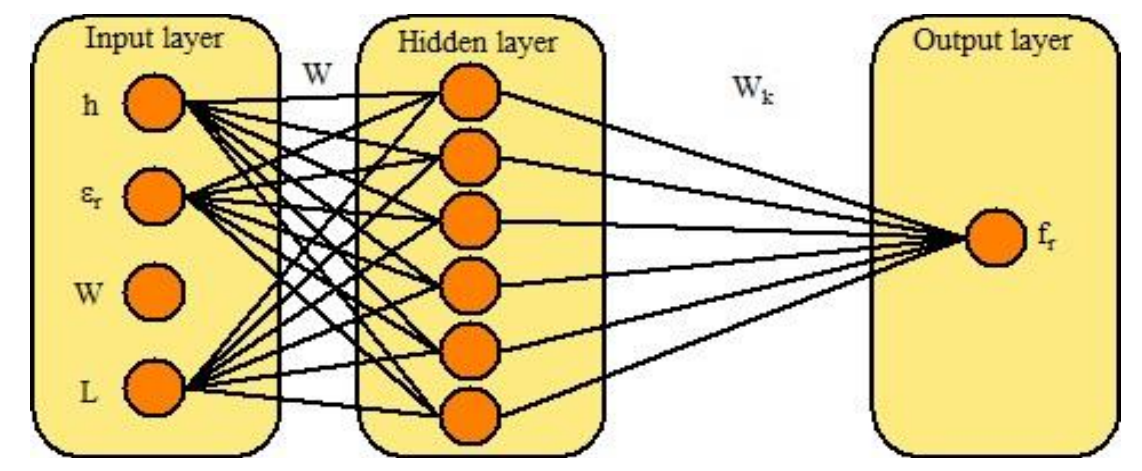

Figure 8. MPA Parametric Verification using ANN

A snippet of the ANN model applied for verification is given in Figure 8. A detailed discussion of the simulated results and allied inferences is given in the subsequent sections.

\section{RESULTS AND DISCUSSION}

In this paper, the predominant emphasis was made on identifying the optimal dimensional parameters of the rectangular MPA antenna model, especially to be used for UWB applications. To achieve it, at first, a patch antenna model was designed with the parameters identified as the height of the dielectric substrate $(\mathrm{h})$, dielectric coefficients also called dielectric constants $\left(\varepsilon_{\mathrm{r}}\right)$, initial cut-off frequency $\left(\mathrm{F}_{\mathrm{r}}\right)$ and Width $(\mathrm{W})$ and Length $(\mathrm{L})$ of the patch antenna. As stated, being knowledge of the data-driven model, at first the training instances were obtained by varying input dimensional parameters while maintaining constant dielectric constants $\left(\varepsilon_{\mathrm{r}}\right)$ and height of the dielectric substrate (h). Concerning the above-stated parameters frequency values were obtained. Thus, inputting the respective values, the $\mathrm{W}$ and $\mathrm{L}$ dimensional parameters were obtained. The results obtained are given in Table 1. 
International Journal of Computer Networks \& Communications (IJCNC) Vol.13, No.3, May 2021

Table 1. Input training samples

\begin{tabular}{|c|c|c|c|c|}
\hline $\mathbf{h}(\mathbf{c m})$ & $\boldsymbol{\varepsilon}_{\mathbf{r}}$ & $\mathbf{W}(\mathbf{c m})$ & $\mathbf{L}(\mathbf{c m})$ & $\mathbf{F}_{\mathbf{r}}(\mathbf{G H z})$ \\
\hline 0.3175 & 2.330 & 5.770 & 3.800 & 2.310 \\
\hline 0.3175 & 2.330 & 4.550 & 3.050 & 2.890 \\
\hline 0.3175 & 2.330 & 2.950 & 1.950 & 4.240 \\
\hline 0.3175 & 2.330 & 1.950 & 1.300 & 5.840 \\
\hline 0.3175 & 2.330 & 1.300 & 1.100 & 6.800 \\
\hline 0.3175 & 2.330 & 1.400 & 0.900 & 7.700 \\
\hline 0.3175 & 2.330 & 1.200 & 0.800 & 8.270 \\
\hline 0.3175 & 2.330 & 1.050 & 0.700 & 9.140 \\
\hline 0.9525 & 2.330 & 1.700 & 1.100 & 4.730 \\
\hline 0.4000 & 2.550 & 0.790 & 1.255 & 7.134 \\
\hline 0.4500 & 2.550 & 0.987 & 1.450 & 6.000 \\
\hline 0.4760 & 2.550 & 1.000 & 1.520 & 5.820 \\
\hline 0.4760 & 2.550 & 0.814 & 1.440 & 6.380 \\
\hline 0.5500 & 2.550 & 0.790 & 1.620 & 5.990 \\
\hline 0.1570 & 2.330 & 0.720 & 1.860 & 5.060 \\
\hline
\end{tabular}

Table 2. ANN-GD based MAP Dimensional estimation

\begin{tabular}{|c|c|c|c|c|}
\hline $\mathbf{h}(\mathbf{c m})$ & $\boldsymbol{\varepsilon}_{\mathbf{r}}$ & $\mathbf{W}(\mathbf{c m})$ & $\mathbf{L}(\mathbf{c m})$ & $\mathbf{F}_{\mathbf{r}}(\mathbf{G H z})$ \\
\hline 0.3175 & 2.330 & 4.984 & 3.811 & 2.907 \\
\hline 0.3175 & 2.330 & 4.830 & 3.201 & 2.237 \\
\hline 0.3175 & 2.330 & 2.266 & 3.142 & 1.643 \\
\hline 0.3175 & 2.330 & 1.569 & 1.306 & 4.625 \\
\hline 0.3175 & 2.330 & 1.620 & 1.087 & 7.067 \\
\hline 0.3175 & 2.330 & 1.490 & 1.005 & 7.039 \\
\hline 0.3175 & 2.330 & 1.256 & 0.792 & 8.090 \\
\hline 0.3175 & 2.330 & 1.042 & 0.767 & 1.000 \\
\hline 0.9525 & 2.330 & 1.812 & 1.119 & 5.631 \\
\hline 0.4000 & 2.550 & 0.880 & 1.105 & 7.351 \\
\hline 0.4500 & 2.550 & 1.002 & 1.400 & 5.943 \\
\hline 0.4760 & 2.550 & 1.214 & 1.498 & 5.522 \\
\hline 0.4760 & 2.550 & 0.911 & 1.390 & 5.583 \\
\hline 0.5500 & 2.550 & 0.980 & 1.626 & 4.900 \\
\hline 0.1570 & 2.330 & 1.920 & 1.799 & 3.550 \\
\hline
\end{tabular}

Table 3. ANN-LM based MAP Dimensional estimation

\begin{tabular}{|c|c|c|c|c|}
\hline $\mathbf{h}(\mathbf{c m})$ & $\boldsymbol{\varepsilon}_{\mathbf{r}}$ & $\mathbf{W}(\mathbf{c m})$ & $\mathbf{L}(\mathbf{c m})$ & $\mathbf{F}_{\mathbf{r}}(\mathbf{G H z})$ \\
\hline 0.3175 & 2.330 & 5.071 & 3.861 & 0.002 \\
\hline 0.3175 & 2.330 & 5.103 & 3.020 & 0.084 \\
\hline 0.3175 & 2.330 & 2.300 & 3.195 & 2.819 \\
\hline 0.3175 & 2.330 & 2.121 & 1.369 & 5.167 \\
\hline 0.3175 & 2.330 & 2.002 & 1.200 & 6.572 \\
\hline 0.3175 & 2.330 & 1.366 & 1.801 & 8.151 \\
\hline 0.3175 & 2.330 & 1.261 & 0.811 & 8.061 \\
\hline 0.3175 & 2.330 & 1.026 & 0.738 & 7.990 \\
\hline 0.9525 & 2.330 & 1.610 & 1.171 & 3.544 \\
\hline 0.4000 & 2.550 & 0.899 & 1.262 & 6.531 \\
\hline 0.4500 & 2.550 & 0.992 & 1.502 & 5.412 \\
\hline 0.4760 & 2.550 & 1.002 & 1.509 & 5.143 \\
\hline 0.4760 & 2.550 & 0.841 & 1.439 & 5.963 \\
\hline 0.5500 & 2.550 & 0.800 & 1.579 & 5.390 \\
\hline 0.1570 & 2.330 & 1.712 & 1.850 & 4.025 \\
\hline
\end{tabular}


International Journal of Computer Networks \& Communications (IJCNC) Vol.13, No.3, May 2021

Table 4. ANN-RBF based MAP Dimensional estimation

\begin{tabular}{|l|c|c|c|c|}
\hline $\mathbf{h}(\mathbf{c m})$ & $\boldsymbol{\varepsilon}_{\mathbf{r}}$ & $\mathbf{W}(\mathbf{c m})$ & $\mathbf{L}(\mathbf{c m})$ & $\mathbf{F}_{\mathbf{r}}(\mathbf{G H z})$ \\
\hline 0.3175 & 2.330 & 5.689 & 3.799 & 1.8758 \\
\hline 0.3175 & 2.330 & 5.455 & 3.011 & 1.2942 \\
\hline 0.3175 & 2.330 & 2.951 & 3.050 & 0.186 \\
\hline 0.3175 & 2.330 & 1.950 & 1.296 & 3.3663 \\
\hline 0.3175 & 2.330 & 1.744 & 1.103 & 6.856 \\
\hline 0.3175 & 2.330 & 1.421 & 0.907 & 7.327 \\
\hline 0.3175 & 2.330 & 1.271 & 0.790 & 8.279 \\
\hline 0.3175 & 2.330 & 1.042 & 0.701 & 8.926 \\
\hline 0.9525 & 2.330 & 1.702 & 1.100 & 8.177 \\
\hline 0.4000 & 2.550 & 0.791 & 1.257 & 5.994 \\
\hline 0.4500 & 2.550 & 0.981 & 1.456 & 5.632 \\
\hline 0.4760 & 2.550 & 1.103 & 1.514 & 5.644 \\
\hline 0.4760 & 2.550 & 0.823 & 1.441 & 6.221 \\
\hline 0.5500 & 2.550 & 0.782 & 1.618 & 6.423 \\
\hline 0.1570 & 2.330 & 1.811 & 1.836 & 2.349 \\
\hline
\end{tabular}

The above-depicted results (Table 2-4) show the dimensional parameters obtained using different ANN variants such as ANN-GD, ANN-LM, and ANN-RBF. Observing overall results and respective inferences, it can be found that a patch antenna with $0.3175 \mathrm{~cm}$ height, $1.042 \mathrm{~cm}$ width, and $0.701 \mathrm{~cm}$ length, in conjunction with the dielectric constant of 2.330 can achieve the highest cut-off frequency of $8.926 \mathrm{GHz}$.

Considering the key issues with the classical ANN-based methods, such as local minima and convergence, in this paper we applied AGA-ANN, where AGA was applied primarily for learning enhancement and dynamic weight estimation. Noticeably, in AGA-ANN implementation for MPA synthesis, we maintained 3 input neurons and 6 hidden neurons, while two neurons were defined as output, to yield two patch's dimensional parameters, W and L. The simulation results obtained by AGA-ANN based model is given in Table 5 .

Table 5. MPA Synthesis results using AGA-ANN

\begin{tabular}{|c|c|c|c|c|}
\hline $\mathbf{h}(\mathbf{c m})$ & $\boldsymbol{\varepsilon}_{\mathbf{r}}$ & $\mathbf{W}(\mathbf{c m})$ & $\mathbf{L}(\mathbf{c m})$ & $\mathbf{F}_{\mathbf{r}}(\mathbf{G H z})$ \\
\hline 0.3175 & 2.330 & 6.779 & 7.141 & 2.310 \\
\hline 0.3175 & 2.330 & 5.868 & 6.297 & 2.890 \\
\hline 0.3175 & 2.330 & 3.652 & 4.160 & 4.240 \\
\hline 0.3175 & 2.330 & 1.526 & 1.849 & 5.840 \\
\hline 0.3175 & 2.330 & 1.277 & 1.539 & 6.800 \\
\hline 0.3175 & 2.330 & 1.178 & 1.408 & 7.700 \\
\hline 0.3175 & 2.330 & 1.080 & 1.271 & 8.270 \\
\hline 0.3175 & 2.330 & 6.922 & 4.845 & 9.140 \\
\hline 0.9525 & 2.330 & 1.144 & 2.158 & 4.730 \\
\hline 0.4000 & 2.550 & 1.163 & 2.180 & 7.134 \\
\hline 0.4500 & 2.550 & 1.170 & 2.187 & 6.000 \\
\hline 0.4760 & 2.550 & 1.158 & 2.171 & 5.820 \\
\hline 0.4760 & 2.550 & 1.176 & 2.171 & 6.380 \\
\hline 0.5500 & 2.550 & 1.171 & 2.181 & 5.990 \\
\hline 0.1570 & 2.330 & 1.165 & 2.031 & 5.060 \\
\hline
\end{tabular}

Observing the results obtained (Table 5), it can be found that amongst the different possible design configurations, the microstrip patch antenna with substrate height of $0.3175 \mathrm{~cm}$, dielectric constant of 2.330, and dimensional characteristics length $4.845 \mathrm{~cm}$, and width of $6.922 \mathrm{~cm}$ 
International Journal of Computer Networks \& Communications (IJCNC) Vol.13, No.3, May 2021

achieves the highest cut-off frequency of $9.140 \mathrm{GHz}$. One very interesting output can be visualized here by observing Table 4 and Table 5. Noticeably, the design parameters by ANNRBF for which the cut-off frequency is the highest (i.e., $8.926 \mathrm{GHz}$ ) are relatively small and hence low cost than the best performance by AGA-ANN. However, since the proposed work doesn't perform physical verification in the form of radiation pattern or SWR, it is must assess whether the performance delivered by the different neuro-computing models is accurate and which should be generalized as the best solution. Considering this fact, we assessed each neurocomputing model for its error-proneness towards prediction. The results of prediction error(s) are given in the subsequent sections.

\subsection{Error Profiling}

Our proposed knowledge-driven model has been assessed in terms of the different error profile parameters such as mean absolute error (MAE), root means square error (RMSE), the mean magnitude of the relative error (MMSE), etc. The performance comparison has been done for the different ANN variants towards MPA design optimization. Before discussing the error profile obtained a snippet of the different error parameters and their respective mathematical models are discussed as follows:

\subsubsection{Mean Absolute Error (MAE)}

Mathematically, MAE is obtained as

$$
\text { MAE }=\frac{1}{n} \sum_{i=1}^{n}\left(\left|Y_{i}^{\prime}-Y_{i}\right|\right)
$$

Where, $Y_{i}^{\prime}$ refers the calculated output, while $Y_{i}$ states for the expected value.

\subsubsection{Mean Magnitude of the Relative Error (MMRE)}

Mathematically, MMRE is obtained as

$$
\text { MMRE }=\frac{1}{n} \sum_{i=1}^{n} \frac{\left|Y_{i}^{\prime}-Y_{i}\right|}{Y_{i}}
$$

Where, $Y_{i}^{\prime}$ signifies the estimate output, while $Y_{i}$ signifies the expected value. To deal with overflow conditions (when denominator becomes Zero $\left(Y_{i}=0\right)$ ), we have added 0.01 in denominator. Thus, MMRE is obtained as

$$
\text { MMRE }=\frac{1}{n} \sum_{i=1}^{n} \frac{\left|Y_{i}^{\prime}-Y_{i}\right|}{Y_{i}+0.01}
$$

\subsubsection{Standard Error of the Mean (SEM)}

Mathematically, SEM is presented as

$$
\mathrm{SEM}=\frac{\sigma}{\sqrt{\mathrm{N}}}
$$


Where, $\sigma$ and $\mathrm{N}$ present the standard deviation and the total number of samples, respectively. The results obtained for the different error parameters are given in Table 6.

Table 6. Error parameters

\begin{tabular}{|c|l|c|c|l|}
\hline Techniques & MAE & MMRE & RMSE & SEM \\
\hline ANN-GD & 0.1190 & 0.2130 & 0.6313 & 0.1120 \\
\hline ANN-RBF & 0.1283 & 0.3545 & 0.1859 & 0.1109 \\
\hline ANN-LM & 0.0078 & 0.0091 & 0.0042 & 0.0873 \\
\hline AGA-ANN & 0.0014 & 0.0045 & 0.00164 & 0.0424 \\
\hline
\end{tabular}

As depicted in Table 6, in comparison to the ANN-GD, ANN-RBF, ANN-LM the proposed AGA-ANN model exhibits minimum average error (0.0014), MMRE (0.0045), RMSE (0.0016), and SEM (0.424). It indicates that the proposed AGA-ANN model can have higher accurate predictions than the other neuro-computing models. This result helps us to conclude or infer that the results of the AGA-ANN model are superior even though it shows higher dimensions than the ANN-RBF model (Table 4). In this reference, exploring the error by ANN-RBF based model, it can be found that it (ANN-RBF) shows higher error (MAE, MMRE, RMSE, and SEM) than the proposed AGA-ANN. This result affirms that the proposed AGA-ANN-based patch antenna design should be considered as the best viable solution than the other approaches, even ANNRBF which shows a smaller size of patch design; however, undergoes higher error proneness. Considering this fact, its resulting outputs (W and $\mathrm{L}$ design parameters) have further been applied for MPA design verification. To perform MPA design verification ANN was modeled with four input neurons, six hidden neurons, and a single output neuron that yielded cut-off-frequency Concerning the dimensional inputs (i.e., $h, \varepsilon_{r}, W$, and $L$ ). The cut-off frequencies for the varied input parameters are given in Table 7.

Table 7. Simulated results for MPA design verification

\begin{tabular}{|c|c|c|c|c|}
\hline $\mathbf{h}(\mathbf{c m})$ & $\boldsymbol{\varepsilon}_{\mathbf{r}}$ & $\mathbf{W}(\mathbf{c m})$ & $\mathbf{L}(\mathbf{c m})$ & $\mathbf{F}_{\mathbf{r}}(\mathbf{G H z})$ \\
\hline 0.3175 & 2.330 & 6.779 & 7.141 & 1.232 \\
\hline 0.3175 & 2.330 & 5.868 & 6.297 & 1.485 \\
\hline 0.3175 & 2.330 & 3.652 & 4.160 & 2.707 \\
\hline 0.3175 & 2.330 & 1.526 & 1.849 & 4.738 \\
\hline 0.3175 & 2.330 & 1.277 & 1.539 & 6.395 \\
\hline $\mathbf{0 . 3 1 7 5}$ & $\mathbf{2 . 3 3 0}$ & $\mathbf{1 . 4 7 8}$ & $\mathbf{1 . 1 0 8}$ & $\mathbf{8 . 2 7 3}$ \\
\hline 0.3175 & 2.330 & 1.080 & 1.271 & 6.990 \\
\hline 0.3175 & 2.330 & 6.922 & 4.845 & 6.640 \\
\hline 0.9525 & 2.330 & 1.144 & 2.158 & 6.300 \\
\hline 0.4000 & 2.550 & 1.163 & 2.180 & 6.236 \\
\hline 0.4500 & 2.550 & 1.170 & 2.187 & 6.021 \\
\hline 0.4760 & 2.550 & 1.158 & 2.171 & 6.001 \\
\hline 0.4760 & 2.550 & 1.176 & 2.171 & 5.912 \\
\hline 0.5500 & 2.550 & 1.171 & 2.181 & 6.023 \\
\hline 0.1570 & 2.330 & 1.165 & 2.031 & 5.342 \\
\hline
\end{tabular}


International Journal of Computer Networks \& Communications (IJCNC) Vol.13, No.3, May 2021

Observing the overall results as depicted in the above tables (Table 6 and Table 7), it can be found that a rectangular MPA with $3.17 \mathrm{~mm}$ of dielectric substrate height $(\mathrm{h})$, and dielectric constant of 2.33 with patch width of $14.78 \mathrm{~mm}$ and a length of $11.08 \mathrm{~mm}$ can achieve the maximum resonant frequency of $8.23 \mathrm{GHz}$. Noticeably, the dimensional parameters of the MPA are small enough to fulfill miniaturization aspects, while delivering a high resonant frequency of $8.23 \mathrm{GHz}$, which can be of great significance for numerous UWB applications such as WLAN, inter-vehicular communication, IoT ecosystems, etc. The overall research conclusion and allied inferences are given in the subsequent sections.

Considering the fact that in a majority of the existing works authors have applied different input design parameters and topological structure and therefore their predicted design parameters roam around the target value. Similarly, in this paper, inputting a set of parameters we intended to obtain the optimal design parameters in sync with some predefined estimated values. Summarily, the predicted output of a model with the different input (data) values would vary from each other, and therefore in this study, an inter-model comparison is not performed. It can also be considered as the motivation for further research, where with obtained design parameters, their respective performance can be assessed in the form of radiation attributes, gain, etc. The overall research conclusion and allied inferences are given in the subsequent sections.

\section{CONCLUSION}

Considering the significance of a robust knowledge -driven approach for rectangular Microstrip patch antenna in this paper the focus was made on obtaining an optimal set of patch dimensional parameters to achieve desired cut-off frequency. To achieve it, this paper inherited the robustness of the software computing environment including data normalization, enhanced machine learning models, heuristic models, etc. Here, the prime objective was to enhance the classical ANN model to yield an optimal set of MPA design parameters for expected cut-off frequency. Also, the implementation of Min-Max normalization enabled alleviating pre-mature saturation and helped to achieve efficient learning over the input data. On the other hand, AGA with adaptive learning ability strengthened ANN to deliver an optimal set of design parameters and respective cut-off frequency. The proposed AGA-ANN based neuron-computational model revealed that for a rectangular MPA with $3.17 \mathrm{~mm}$ of dielectric height $(\mathrm{h})$, a dielectric constant of 2.33 and, 14.78 $\mathrm{mm}$ and $11.08 \mathrm{~mm}$ of width and length, respectively can achieve the maximum resonant frequency of $8.23 \mathrm{GHz}$, which can be of utmost significance for low-size compact MPA for UWB applications. Though the proposed model exhibited better solution with generic expectations of compact size (miniaturized) and predicted cut-off frequency, it could employ merely 15 sets of instances to perform training. Recalling the fact that increasing the numerous of training instances can yield more accurate and reliable performance. In the future more efficient computing environment such as up-sampling, feature selection, and hidden neuron adaptive neuron-computational model can be developed. Additionally, performance can also be assessed for its under-fitting or over-fitting conditions to yield the most reliable and efficient MPA design solution. Though, the results obtained signify the optimality of the proposed rectangular microstrip patch antenna design for UWB applications; however, its realistic behavior voltage standing wave ratio (VSWR), gain and radiation pattern could not be assessed. Moreover, literature indicates that poly lines structure or other Omni-directional pattern- centric designs can have higher coverage and signal performance. These key factors can be considered as the future scope for further study.

\section{CONFLICTS OF INTEREST}

The authors declare no conflict of interest. 
International Journal of Computer Networks \& Communications (IJCNC) Vol.13, No.3, May 2021

\section{REFERENCES}

[1] Pozar, D. M. (1992) “Microstrip Antennas”,IEEE proc., Vol. 80, No.1, pp. 79-91.

[2] Orban, D.,\&Moernaut, G.J.K. (2009) “The Basics of Patch Antennas”, www. rfglobalnet. Com

[3] Singh, I.,\&Tripathi, V.S. (2011) "Micro strip Patch Antenna and its Applications: A Survey",Int. J. Comp. Tech. Appl., Vol. 2, No. 5, pp.1595-1599.

[4] Balanis, C. A. (2005) “Antenna Theory, Analysis and Design” John Wiley \& Sons, New York.

[5] Cakir, G.,\&Sevgi, L. (2005) "Design, Simulation and Tests of a Low cost Microstrip Patch Antenna Arrays for the Wireless Communication”,Turk J. Elec. Engineering, Vol. 13, No.1, pp. 93-103.

[6] Ozkayaa, U. (2015) "Dimensions Optimization of Microstrip Patch Antenna in X/Ku Band via Artificial Neural Network”, Elsevier, Vol. 195, pp.2520-2526.

[7] Kala, P.,Saxena, R.,kumar, M.,KumarA.,\&Pant, R. (2012) "Design Of Rectangular Patch Antenna Using MLP Artificial Neural Network”, Journal of Global Research in Computer Science, Vol. 3, No. 5, pp.11-14.

[8] Santos, F. N. d.,Nascimento, S. S.,Esquerre, V. F. R.,\&Filho, F. G. S. (2011, November) "Analysis and Design of Microstrip Antennas by Artificial Neural Networks",International Conference on Microwave \& Optoelectronics Conference, IEEE, pp.226-230.

[9] Sivia, J. S. (2016) "Neuron-computational Models for Parameter Estimation of Circular Microstrip Patch Antennas",ProcediaComputer Science, Elsevier, Vol. 85, pp. 393-400.

[10] Karimabadi, S. S.,Mohsenzadeh, Y.,Attari, A. R.,\&Moghadasi, S. M. (2008) "Bandwidth Enhancement of Single feed Circularly Polarized Equilateral Triangular Microstrip Antenna",Progress In Electromagnetics Research Symposium, pp. 147-150.

[11] Kushwah, V. S., \&Tomar, G. S. (2011,June) "Size reduction of Microstrip Patch Antenna using Defected Microstrip Structures",International Conf. on Communication Systems and Network Technologies,pp. 203 -207.

[12] Kaur, R., \&Narula, A. K. (2017) “Artificial Neural Network based Design of Modified Shaped Patch Antenna",I.J. Intelligent Systems and Applications, Vol.4, pp. 32-38.

[13] Rakhi, \&Thakur, S. (2014) "Performance Analysis of Different Shapes Patch Antennas at 2.45 GHz”, International Journal of Engineering Research and Technology, Vol.3, Issue 9, pp. 83-86.

[14] Thakare,V.,\&Singhal, P. K. (2009) "Bandwidth Analysis by introducing slots in microstrip antenna design using ANN",Progress In Electromagnetic Research M, Vol. 9, pp. 107-122.

[15] Neog, D. K.,Pattnaik, S. S.,Panda, D. C.,Devi, S.,KhuntiaB., \&Dutta, M. (2005) "Design of a Wideband Microstrip Antenna and the Use of Artificial Neural Networks in Parameter Calculation",IEEE Antennas and Prop. Magazine, Vol. 47, No.3, pp-60-65.

[16] Mishra, A., Nandgaonkar, A.B.,BhagileV. D.,Mehrotra,S. C.,\&Patil, P.M. (2009, December)“Design of Square and Rectangular Microstrip Antenna with the use of FFBP algorithm of Artificial Neural Network",Applied Electromagnetics Conference, pp. 1-4.

[17] Turker, N.,GunesF., \&Yildirim, T. (2006) “Artificial Neural Design of microstrip Antennas",Turk J Elec. Engineering, Vol. 14, No.3, pp. 445-453.

[18] Merad, L.,Merad, L.,Bendimerad ,F. T.,\&Meriah S. M. (2011) "Design and resonant frequency calculation of rectangular microstrip antennas",International Journal of Numerical Modelling Electronic Networks Devices and Fields, Vol. 24, No. 2, pp. 144-153.

[19] Manh, L. H.,Grimaccia, F., Mussetta, M.,Pirinoli, P., \&Zich, R.E. (2014, August) "Multi-Net ANN approach for improving antenna optimization",International Conference on General Assembly and Scientific Symposium, IEEE, pp.1-4.

[20] Wang, Z.,Fang, S.,Wang, Q., \&Liu, H. (2012) “An ANN-Based Synthesis Model for the Single-Feed circularly Polarized Square Microstrip Antenna with Truncated Corners",IEEE Transactions on antennas and propagation, Vol. 60, No. 12, pp. 5989 - 5992.

[21] Tighilt, Y.,Bouttout, F.,\&Khellaf, A. (2011) "Modeling and Design of Printed Antennas Using Neural Networks",International Journal of RF and Microwave Comp.-Aided Engineering, Vol. 21, Issue2, pp. 228-233.

[22] Can, S.,Kapusuz, K. Y.,\&Aydin, E. (2013) "Neural Network Based Resonant Frequency Solver For Rectangular-Shaped Shorting Pin-Loaded Antennas",Microwave and Optical Technology Letters, Vol. 55, Issue12, pp. 3025-3028.

[23] Freni, A.,Mussetta, M.,\&Pirinoli, P. (2012) "Neural network characterization of reflectarray antennas",Int. J. Antennas Propagation, Article ID 541354, pp. 1-10. 
International Journal of Computer Networks \& Communications (IJCNC) Vol.13, No.3, May 2021

[24] Patnaik, A.,Mishra, R. K.,Patra, G. K.,\&Dash, S. K. (1997) “An Artificial Neural Network Model for Effective Dielectric Constant of Microstrip Line",IEEE Trans. on Antennas and Prop., Vol. 45, No. 11, pp. 1697.

[25] Gultekin, S.,Guney, K.,\&Sagiroglu, S. (2001) “A Comparative study of Neural Networks for Resonant Frequency Computation of Electrically Thin and Thick Circular Microstrip Antenna",International Conference on Electrical and Electronics Engineering, Turkey, pp. 204-208.

[26] Gupta, V. R., \&Gupta, N.(2005) "An Artificial Neural Network Model for Feed Position of the Microstrip Antenna”,ElektronikairElektrotechnika, Vol. 60, No. 4, pp. 87-89.

[27] Khan ,T., De, A., \&Uddin , M.( 2013)"Prediction of Slot-Size and Inserted Air Gap for Improving the Performance of Rectangular Microstrip Antennas Using Artificial Neural Networks", IEEE Antennas And Wireless Propagation Letters, Vol. 12, pp. 1367-1371.

[28] Thakare ,V. V., \&Singhal, P. K.(2010) "Neural Network Based CAD Model For the design of Rectangular Patch Antenna", Journal of Engineering and Technology Research, Vol. 2, pp. 126-129.

[29] Thakare,V. V. \&Singhal, P. K.,(2010) "Estimation of Input Impedance of Microstrip Patch Antenna Using Fuzzy Neural Network”,International Journal of Engineering Science and Technology, Vol. 2, No.10, pp. 5497-5503.

[30] Heidari,A. A.,\&Dadgarnia, A.(2011, August) "Design and Optimization of a Circularly Polarized Microstrip Antenna for GPS Applications Using ANFIS and GA", $32^{\text {th }}$ URSI General Assembly and Scientific Symposium, pp. 1-4.

[31] Hamdy, S.,Khouly, A. E.,ZakiA.,\&Khamy, S. E. E.(2016,August) "A new fractal-like tree structure of circular patch antennas for 5G multi-band and wide-band applications",Progress in Electromagnetic Research Symposium, pp. 2467.

[32] Halawani, M. G., Najjar, A.- M. A., \&Abdelazeez, M. K.(2016, July) "Microstrip antenna design for UWB applications",IEEE International Symposium on Antennas and Propagation, pp. 43-44.

[33] Chakradhar, K. S.,Rao,P. M.,\&Rao,B. R.(2017, September) "Design of a compact dumbbell shape micro strip patch antenna for UWB applications",IEEE International Conference on Power, Control, Signals and Instrumentation Engineering, pp. 2854-2857.

[34] Mishra,R.,Kalyan,R., \&Dubey,Y. M. (2017, April) "Miniaturized W slot ultrawide band microstrip antenna for short distance communication",International conference of Electronics, Communication and Aerospace Technology, pp. 332-336.

[35] Khan,S.,Nasir, H. J.,Sieler, L.,\&Tanougast,C. (2018, June) “A U-shaped Wide band frequency reconfigurable dielectric resonator antenna for 5G applications",International Symposium on Networks, Computers and Communications, pp. 1-4.

[36] Roy ,C. S.,\& Roy, P. S. (2015, March) "Design of slotted Microstrip patch Antenna with enhanced bandwidth in C- band applications",IEEE International Conference on Electrical, Computer and Communication Technologies, pp. 1-4.

[37] Motevasselian,A., \&Whittow,W. G.(2017) "Miniaturization of aAntenna Using an Arc Projection",IEEE Antennas and Wireless Propagation Letters, Vol. 16,pp. 517-520.

[38] Hasan, R.,Ahmed,W. A.,Lu,J.,Ng, H. J.,\&Kissinger, D.(2019,August) “\$F\$ -Band Differential Microstrip Patch Antenna Array and Waveguide to Differential Microstrip Line Transition for FMCW Radar Sensor”,IEEE Sensors Journal, Vol. 19, No. 15, pp. 6486-6496.

[39] Radavaram,S.,\& Pour, M.(2019) "Wideband Radiation Reconfigurable Microstrip Patch Antenna Loaded With Two Inverted U-Slots”, IEEE Transaction on Antennas and Propagation, Vol. 67, No. 3, pp. 1501-1508.

[40] Zhang,J.,Zhu,L.,Wu,Q.,Liu,N.,\&Wu,W.(2016) “A Compact Microstrip-Fed Patch Antenna With Enhanced Bandwidth and Harmonic Suppression", IEEE Transaction on Antennas and Propagation, Vol. 64, No.12, pp. 5030-5037.

[41] Zang,Y.,Zhai, H.,Xi ,L.,\&Li, L.(2019) “A Compact Microstrip Antenna With Enhanced Bandwidth and Ultra-Wideband Harmonic Suppression”,IEEE Transaction on Antennas and Propagation, Vol. 67, No.3, pp.1969-1974.

[42] Jafargholi, A., Jafargholi, A.,\&Ghalamkari, B.(2018) "Dual-Band Slim Microstrip Patch Antennas",IEEE Transactions on Antennas and Propagation, Vol. 66, No. 12, pp. 6818-6825.

[43] Liu,S.,Wu,W.,\& Fang,D.(2016) "Wideband Monopole-Like Radiation Pattern Circular Patch Antenna with High Gain and Low Cross-Polarization”, IEEE Transactions on Antennas and Propagation, Vol. 64, No. 5, pp. 2042-2045. 
International Journal of Computer Networks \& Communications (IJCNC) Vol.13, No.3, May 2021

[44] He,Y.,Li,Y., Sun, W., Zhang ,Z.,\&Chen, P. (2018) "Dual Linearly Polarized Microstrip Antenna Using a Slot-Loaded TM50Mode",IEEE Antennas and Wireless Propagation Letters, Vol. 17, No. 12, pp. 2344-2348.

[45] Asaadi,M., \&Sebak, A. (2017) "Gain and Bandwidth Enhancement of $2 \times 2$ Square Dense Dielectric Patch Antenna Array Using a Holey Superstrate”, IEEE Antennas and Wireless Propagation. Letters, Vol. 16, pp. 1808-1811.

[46] Gao, S.,Ge, L.,Zhang, D.,\&. Qin, W. (2018) "Low-Profile Dual-Band Stacked Microstrip Monopolar Patch Antenna for WLAN and Car-to-Car Communications",IEEE Access, Vol. 6, pp. 69575-69581.

[47] Chen, Q.,Li, J.,Yang, G., Cao, B.,\& Zhang, Z.,(2019) “A Polarization-Reconfigurable High-Gain Microstrip Antenna",IEEE Transactions on Antennas and Propagation, Vol. 67, No. 5, pp. 34613466. .

[48] Khan, T.,De, A.,\&Uddin, M. (2013) "Prediction of Slot-Size and Inserted Air-Gap for Improving the Performance of Rectangular Microstrip Antennas Using Artificial Neural Networks",IEEE Antennas and Wireless Propagation Letters, Vol. 12, pp. 1367-1371.

[49] Mishra,R. K.,\&Patnaik, A. (2003) "Designing rectangular patch antenna using the neurospectral method", IEEE Transactions on Antennas and Propagation, Vol. 51, No. 8, pp. 1914-1921.

[50] Kaur, A.,\& Mittal, R. (2018, June) "Dimensions Estimation of Wide Band Rectangular Microstrip Patch Antenna Based on Artificial Neural Network", $2^{\text {nd }}$ International Conference on Intelligent Computing and Control Systems, pp. 1644-1648.

[51] Neebha,T. M.,\&Nesasudha, M. (2017, March) "Artificial neural network based design of a microstrip patch antenna for RADAR applications", $1^{\text {st }}$ International Conference on Recent Advances in Aerospace Engineering, pp. 1-6.

[52] Singh, B. K. (2015,February) "Design of rectangular microstrip patch antenna based on Artificial Neural Network algorithm”, $2^{\text {nd }}$ International Conference on Signal Processing and Integrated Networks, pp. 6-9.

[53] Singh, J.,Singh, G.,Kaur, S.,\&Sohi, B. S.(2015,December) "Performance analysis of different neural network models for parameters estimation of coaxial fed $2.4 \mathrm{GHz}$ E-shaped Microstrip patch antenna", $2^{\text {nd }}$ International Conference on Recent Advances in Engineering \& Computational Sciences, pp. 1-5.

[54] Nayak,C.,\&Kumar, P. (2017, September) “Optimized Design and Analysis of Microstrip Antenna using Artificial Neural Network",International Conference on Current Trends in Computer, Electrical, Electronics and Communication, pp. 114-118.

[55] Deshmukh,A. A..Venkata A. P. C., Nagarbowdi,S.,\&Kulkarni, S. D. (2015, January) “Artificial Neural Network model for suspended equilateral triangular microstrip antennas”, International Conference on Communication, Information \& Computing Technology, pp. 1-4.

[56] Jain,S. K.(2015, February) "Size reduction of stacked patch antenna using neural network based swarm Optimizer",National Conference on Recent Advances in Electronics \& Computer Engineering,pp. 170-175.

[57] Chetioui,M., Boudkhil, A.,Benabdallah, N.,\&Benahmed,N. (2018, April) "Design and optimization of SIW patch antenna for Ku band applications using ANN algorithms", $4^{\text {th }}$ International Conference on Optimization and Applications, pp. 1-4.

[58] Varma, R.,\&Ghosh,J.(2016, March) "Design of slot loaded proximity coupled microstrip antennas using knowledge based neural networks", $3^{\text {rd }}$ International Conference on Recent Advances in Information Technology, pp. 209-214.

[59] Yoo, S., Milyakh, Y., Kim H., Hong, C. \&Choo, H. (2020) "Patch Array Antenna Using a Dual Coupled Feeding Structure for $79 \mathrm{GHz}$ Automotive Radar Applications", IEEE Antennas and Wireless Propagation Letters, Vol. 19, No. 4, pp. 676-679.

[60] Bondarik, A. \&Sjöberg, D. (2020) "Gridded parasitic patch stacked microstrip array antenna for $60 \mathrm{GHz}$ band”, IET Microwaves, Antennas \& Propagation, Vol. 14, No. 8, pp. 712-717.

[61] Sun. S. (2019) "A Design of Compact Ultrawideband Circularly Polarized Microstrip Patch Antenna", IEEE Transactions on Antennas and Propagation, Vol. 67, No. 9, pp. 6170-6175.

[62] Liu,Z. X., Zhu, L.,\& Liu, N.-W. (2020) "A Compact Omnidirectional Patch Antenna with Ultrawideband Harmonic Suppression", IEEE Transactions on Antennas and Propagation, Vol. 68, No. 11, pp. 7640-7645.

[63] C., Sun (2020) "A Design of Low Profile Microstrip Patch Antenna with Bandwidth Enhancement”, IEEE Access, Vol. 8, pp. 181988-181997. 
International Journal of Computer Networks \& Communications (IJCNC) Vol.13, No.3, May 2021

[64] Xu, J., Hong, W., Jiang, Z. H., \& Zhang, H. (2019) "Wideband, Low-Profile Patch Array Antenna with Corporate Stacked Microstrip and Substrate Integrated Waveguide Feeding Structure", IEEE Transactions on Antennas and Propagation, Vol. 67, No. 2, pp. 1368-1373.

[65] Khan, S., Daiya, V., Ebenezer, J.,\&Jehadeesan, R. (2019) "Novel Patch Antenna Design for Wireless Channel Monitors", 10th International Conference on Computing, Communication and Networking Technologies (ICCCNT), India, pp. 1-6.

[66] Shen, X., Liu, Y., Zhao, L., Huang, G., Shi, X., \& Huang, Q. (2019) “A Miniaturized Microstrip Antenna Array at 5G Millimeter-Wave Band", IEEE Antennas and Wireless Propagation Letters, Vol. 18, No. 8, pp. 1671-1675.

[67] Shahidul, I. M., Islam, M. T., Ullah, M. A., Kok,Beng G., Amin, N.,\& Misran, N., (2019)“A Modified Meander Line Microstrip Patch Antenna with Enhanced Bandwidth for $2.4 \mathrm{GHz}$ ISM-Band Internet of Things (IoT) Applications", IEEE Access, Vol. 7, pp. 127850-127861.

[68] Chemkha, H.,\&Belkacem, A., (2020)“Design of New Inset Fed Rectangular Microstrip Patch Antenna with Improved Fundamental Parameters”, 2020 IEEE International Conference on Design \& Test of Integrated Micro \& Nano-Systems (DTS), Tunisia, pp. 1-4.

[69] Hamid, S. H., Chin, H. G., Kiong, T. S.,\&Ferdous, N., (2019) "Microstrip Patch Antenna Design in Circular Topology for Ultra High-Frequency 900MHz Radio Spectrum: Size Reduction Technique and Defected Ground Structure Effects", IEEE Conference on Sustainable Utilization and Development in Engineering and Technologies (CSUDET), Malaysia, pp. 271-275.

[70] Aneesh, Md., Singh, A., Kamakshi, K., \& Ansari, J. A. (2019) "Performance Investigations of Sshaped RMSA Using Multilayer Perceptron Neural Network for S-Band Applications",Radioelectronics and Communications Systems, Vol. 62, No. 8, pp. 400-408.

[71] Siddiqui, Md. G., Saroj, A. K., Devesh, Saxena, R., Ansari, J. A., \&Sayeed, S. S. (2018,November) "Soft Computing based Ellipsoidal Fractal Antenna for Wide Band Applications", 3rd International Innovative Applications of Computational Intelligence on Power, Energy and Controls with their Impact on Humanity (CIPECH), India,DOI: 10.1109/CIPECH.2018.8724161.

[72] Singh, V. K., Lala, A., \& Singh, A. K. (2020) "Novel Inset Feed Circular Slotted Microstrip Antenna Using Multilayer Feed-Forward Back-Propagation and Radial Basis Function Neural Network",National Academy Science Letters, Vol. 43, pp.343-345. 